(C) 2004 American Chemical Society, J. Am. Chem. Soc., Timoshkin ja0400483 Supporting Info Page 1

Supplementary Materials.

Table 1S. Prediction of the standard enthalpy, $\mathrm{kJ} \mathrm{mol}^{-1}$ of formation for $\mathrm{GaH}_{3}(\mathrm{~g})$.

\begin{tabular}{l|cc}
\hline Process & $\begin{array}{c}\Delta \mathrm{H}^{0}{ }_{298,} \\
\mathrm{CCSD}(\mathrm{T}) / \mathrm{pVTZ}\end{array}$ & $\begin{array}{c}\Delta_{\mathrm{f}} \mathrm{H}^{0}{ }_{298} \\
\left(\mathrm{GaH}_{3(\mathrm{~g})}\right)\end{array}$ \\
\hline $\mathrm{GaH}_{3}=\mathrm{Ga}+3 \mathrm{H}$ & 812.7 & 114.2 \\
$\mathrm{GaH}_{3}=\mathrm{Ga}+{ }^{3} /{ }_{2} \mathrm{H}_{2}$ & 166.5 & 106.4 \\
$\mathrm{GaH}_{3}=\mathrm{GaH}+\mathrm{H}_{2}$ & 95.5 & 119.8 \\
& mean & 113.4 \\
\hline
\end{tabular}

The following formation enthalpies have been used:

$\Delta_{\mathrm{f}} \mathrm{H}^{0}{ }_{298}\left(\mathrm{H}_{(\mathrm{g})}\right)=2118.0 \mathrm{~kJ} \mathrm{~mol}^{-1} ; \Delta_{\mathrm{f}} \mathrm{H}^{0}{ }_{298}\left(\mathrm{GaH}_{(\mathrm{g})}\right)=215.3 \mathrm{~kJ} \mathrm{~mol}^{-1} ; \Delta_{\mathrm{f}} \mathrm{H}^{0}{ }_{298}\left(\mathrm{Ga}_{(\mathrm{g})}\right)=$ $272.9 \mathrm{~kJ} \mathrm{~mol}^{-1}$. 
(C) 2004 American Chemical Society, J. Am. Chem. Soc., Timoshkin ja0400483 Supporting Info Page 2

Table 2S. Harmonic Vibrational frequencies $\omega, \mathrm{cm}^{-1}$, and IR intencities I, $\mathrm{km} \mathrm{mol}^{-1}$, for imidocompounds. B3LYP/pVDZ level of theory, unscaled.

\begin{tabular}{|c|c|c|c|c|c|c|}
\hline & $\omega$ & $I$ & $\omega$ & I & $\omega$ & I \\
\hline \multirow{26}{*}{$\overline{[\mathrm{HGaNH}]_{7} \mathrm{C}_{3 \mathrm{v}}}$} & 94.7 & 0.000 & 103.4 & 0.006 & 103.4 & 0.006 \\
\hline & 130.2 & 0.138 & 130.2 & 0.139 & 135.2 & 0.121 \\
\hline & 182.8 & 0.139 & 182.8 & 0.139 & 207.7 & 0.018 \\
\hline & 207.7 & 0.018 & 221.7 & 0.328 & 221.7 & 0.329 \\
\hline & 230.3 & 0.332 & 279.8 & 1.389 & 294.2 & 1.369 \\
\hline & 368.6 & 0.197 & 368.6 & 0.197 & 391.0 & 0.002 \\
\hline & 391.0 & 0.002 & 403.0 & 0.069 & 403.0 & 0.070 \\
\hline & 414.9 & 0.000 & 437.6 & 6.335 & 443.1 & 0.000 \\
\hline & 466.4 & 0.007 & 466.4 & 0.007 & 478.0 & 1.099 \\
\hline & 478.0 & 1.102 & 497.8 & 64.838 & 532.4 & 0.045 \\
\hline & 540.5 & 18.757 & 540.5 & 18.753 & 548.4 & 16.029 \\
\hline & 548.4 & 16.024 & 566.8 & 0.000 & 572.5 & 1.164 \\
\hline & 572.5 & 1.164 & 593.6 & 0.000 & 637.7 & 493.279 \\
\hline & 637.7 & 493.148 & 639.6 & 431.681 & 641.3 & 209.522 \\
\hline & 643.4 & 65.430 & 643.4 & 65.217 & 656.1 & 239.067 \\
\hline & 661.4 & 26.224 & 661.4 & 26.239 & 679.2 & 15.095 \\
\hline & 679.2 & 15.094 & 706.5 & 79.686 & 825.7 & 60.528 \\
\hline & 825.7 & 60.538 & 831.0 & 20.901 & 831.0 & 20.879 \\
\hline & 868.2 & 213.851 & 890.2 & 0.000 & 913.3 & 0.000 \\
\hline & 923.5 & 101.575 & 923.5 & 101.624 & 954.5 & 80.598 \\
\hline & 954.5 & 80.647 & 1001.8 & 36.505 & 1001.9 & 36.500 \\
\hline & 1012.3 & 149.352 & 1939.5 & 348.650 & 1939.5 & 348.556 \\
\hline & 1945.2 & 65.252 & 1978.8 & 299.819 & 1978.8 & 299.842 \\
\hline & 1987.2 & 132.849 & 2001.4 & 208.430 & 3480.3 & 4.040 \\
\hline & 3481.1 & 18.448 & 3481.1 & 18.445 & 3541.0 & 8.609 \\
\hline & 3543.1 & 19.498 & 3543.1 & 19.518 & 3566.8 & 16.741 \\
\hline \multirow[t]{17}{*}[\mathrm{HGaNH}]{$_{8} \mathrm{~S}_{4}$} & 96.3 & 0.549 & 97.9 & 0.111 & 97.9 & 0.111 \\
\hline & 118.5 & 0.000 & 128.0 & 0.053 & 133.5 & 0.000 \\
\hline & 138.7 & 0.050 & 138.7 & 0.050 & 160.4 & 0.001 \\
\hline & 198.8 & 0.000 & 204.5 & 0.075 & 208.3 & 0.486 \\
\hline & 208.3 & 0.486 & 224.5 & 0.000 & 244.6 & 0.388 \\
\hline & 244.6 & 0.388 & 259.3 & 0.000 & 277.7 & 1.504 \\
\hline & 355.3 & 0.000 & 363.7 & 0.004 & 381.5 & 0.398 \\
\hline & 381.5 & 0.398 & 390.2 & 1.347 & 391.1 & 1.536 \\
\hline & 391.1 & 1.536 & 424.6 & 0.000 & 434.6 & 0.000 \\
\hline & 442.1 & 0.582 & 442.1 & 0.582 & 448.7 & 0.000 \\
\hline & 466.0 & 8.277 & 466.0 & 8.277 & 507.4 & 0.000 \\
\hline & 511.4 & 42.449 & 528.3 & 22.992 & 528.3 & 22.992 \\
\hline & 542.0 & 0.000 & 551.4 & 0.000 & 555.9 & 2.798 \\
\hline & 566.4 & 13.470 & 566.4 & 13.470 & 567.3 & 0.000 \\
\hline & 576.9 & $1: 453$ & 625.0 & 7.324 & 625.0 & 7.324 \\
\hline & 633.7 & 300.971 & 633.7 & 300.971 & 645.4 & 800.082 \\
\hline & 648.1 & 0.000 & 653.6 & 283.247 & 656.5 & 389.048 \\
\hline
\end{tabular}


(C) 2004 American Chemical Society, J. Am. Chem. Soc., Timoshkin ja0400483 Supporting Info Page 3

\begin{tabular}{|c|c|c|c|c|c|c|}
\hline & 656.5 & 389.048 & 665.8 & 0.000 & 673.4 & 18.950 \\
\hline & 701.4 & 71.353 & 701.4 & 71.353 & 702.1 & 76.591 \\
\hline & 706.4 & 0.000 & 817.9 & 101.953 & 817.9 & 101.954 \\
\hline & 821.3 & 46.655 & 821.5 & 0.000 & 900.0 & 0.000 \\
\hline & 903.3 & 72.454 & 904.9 & 74.026 & 904.9 & 74.026 \\
\hline & 952.7 & 99.415 & 952.7 & 99.415 & 967.3 & 167.651 \\
\hline & 969.5 & 0.000 & 1008.0 & 0.000 & 1019.0 & 64.686 \\
\hline & 1019.0 & 64.686 & 1025.8 & 98.135 & 1936.2 & 7.035 \\
\hline & 1938.0 & 473.980 & 1938.0 & 473.979 & 1942.0 & 0.000 \\
\hline & 1976.7 & 252.228 & 1976.7 & 252.228 & 1980.0 & 548.951 \\
\hline & 1984.4 & 0.000 & 3478.9 & 0.000 & 3479.3 & 30.163 \\
\hline & 3479.3 & 30.163 & 3479.8 & 0.921 & 3530.3 & 38.807 \\
\hline & 3530.3 & 0.000 & 3531.3 & 15.722 & 3531.3 & 15.722 \\
\hline \multirow{34}{*}[\mathrm{HGaNH}]{$_{9} \mathrm{C}_{3 \mathrm{~h}}$} & 100.7 & 0.000 & 100.7 & 0.000 & 105.3 & 0.104 \\
\hline & 105.3 & 0.104 & 117.5 & 0.000 & 122.2 & 0.361 \\
\hline & 125.8 & 0.186 & 125.8 & 0.186 & 140.6 & 0.000 \\
\hline & 140.6 & 0.000 & 176.1 & 0.000 & 199.6 & 0.257 \\
\hline & 211.2 & 0.580 & 211.2 & 0.579 & 218.0 & 0.000 \\
\hline & 218.0 & 0.000 & 221.7 & 0.913 & 222.4 & 0.000 \\
\hline & 246.2 & 0.000 & 270.6 & 1.481 & 270.6 & 1.480 \\
\hline & 358.4 & 0.158 & 358.4 & 0.159 & 365.7 & 0.000 \\
\hline & 365.7 & 0.000 & 377.4 & 0.000 & 394.5 & 0.138 \\
\hline & 404.6 & 3.615 & 404.6 & 3.614 & 425.9 & 0.028 \\
\hline & 428.0 & 0.000 & 438.0 & 0.000 & 438.0 & 0.000 \\
\hline & 458.2 & 0.000 & 477.4 & 25.750 & 477.4 & 25.752 \\
\hline & 486.9 & 0.000 & 486.9 & 0.000 & 516.9 & 0.319 \\
\hline & 526.0 & 0.000 & 529.3 & 24.507 & 529.3 & 24.513 \\
\hline & 557.7 & 0.000 & 560.8 & 8.517 & 560.8 & 8.518 \\
\hline & 565.3 & 0.000 & 565.3 & 0.000 & 581.4 & 12.684 \\
\hline & 581.8 & 0.000 & 621.0 & 7.370 & 622.4 & 0.000 \\
\hline & 622.4 & 0.000 & 643.0 & 492.754 & 643.0 & 492.791 \\
\hline & 647.8 & 0.000 & 651.4 & 973.021 & 658.7 & 450.083 \\
\hline & 658.7 & 450.103 & 665.7 & 0.000 & 665.7 & 0.000 \\
\hline & 703.4 & 0.000 & 703.4 & 0.000 & 706.8 & 0.000 \\
\hline & 707.5 & 179.255 & 707.5 & 179.263 & 710.5 & 0.487 \\
\hline & 832.2 & 0.000 & 833.4 & 93.255 & 833.4 & 93.253 \\
\hline & 890.5 & 0.000 & 890.5 & 0.000 & 901.7 & 58.643 \\
\hline & 913.9 & 132.943 & 913.9 & 132.946 & 919.3 & 0.000 \\
\hline & 946.7 & 0.000 & 946.7 & 0.000 & 952.4 & 244.626 \\
\hline & 1017.7 & 44.956 & 1024.1 & 0.000 & 1031.9 & 0.000 \\
\hline & 1031.9 & 0.000 & 1034.9 & 148.029 & 1034.9 & 148.012 \\
\hline & 1939.8 & 0.000 & 1939.8 & 0.000 & 1941.9 & 287.373 \\
\hline & 1941.9 & 287.356 & 1943.3 & 935.206 & 1948.7 & 0.000 \\
\hline & 1975.3 & 398.235 & 1975.3 & 398.197 & 1979.1 & 0.000 \\
\hline & 3477.6 & 0.000 & 3477.9 & 22.795 & 3477.9 & 22.783 \\
\hline & 3478.0 & 54.089 & 3478.5 & 0.000 & 3478.5 & 0.000 \\
\hline & 3530.9 & 26.612 & 3530.9 & 26.618 & 3531.0 & 0.000 \\
\hline$[\mathrm{HGaNH}]_{10} \mathrm{C}_{3 \mathrm{v}}$ & 66.4 & 0.004 & 66.4 & 0.004 & 73.8 & 0.000 \\
\hline Needle-shaped & 101.2 & 0.113 & 111.3 & 0.000 & 115.8 & 0.011 \\
\hline
\end{tabular}


(C) 2004 American Chemical Society, J. Am. Chem. Soc., Timoshkin ja0400483 Supporting Info Page 4

\begin{tabular}{|c|c|c|c|c|c|c|}
\hline & 115.8 & 0.011 & 122.6 & 0.025 & 122.6 & 0.025 \\
\hline & 139.6 & 0.002 & 139.6 & 0.002 & 174.2 & 1.404 \\
\hline & 184.1 & 0.088 & 184.1 & 0.088 & 204.9 & 0.076 \\
\hline & 204.9 & 0.076 & 215.3 & 0.324 & 215.3 & 0.324 \\
\hline & 225.3 & 0.019 & 225.3 & 0.018 & 236.1 & 0.317 \\
\hline & 247.4 & 0.968 & 285.0 & 0.575 & 291.6 & 1.907 \\
\hline & 373.6 & 0.133 & 373.6 & 0.133 & 387.9 & 0.056 \\
\hline & 387.9 & 0.056 & 391.4 & 0.633 & 391.4 & 0.634 \\
\hline & 399.3 & 0.550 & 399.3 & 0.550 & 425.1 & 0.000 \\
\hline & 435.0 & 0.000 & 445.7 & 8.229 & 451.6 & 21.313 \\
\hline & 466.6 & 0.000 & 467.2 & 0.002 & 467.2 & 0.002 \\
\hline & 483.1 & 2.321 & 483.1 & 2.325 & 498.2 & 0.195 \\
\hline & 498.2 & 0.195 & 512.2 & 84.242 & 520.2 & 35.314 \\
\hline & 543.0 & 8.397 & 543.0 & 8.401 & 551.4 & 34.452 \\
\hline & 551.4 & 34.455 & 561.3 & 4.902 & 561.3 & 4.895 \\
\hline & 575.0 & 0.000 & 591.1 & 0.000 & 597.5 & 4.253 \\
\hline & 597.5 & 4.253 & 600.1 & 246.649 & 621.4 & 0.672 \\
\hline & 621.4 & 0.674 & 634.9 & 1128.379 & 640.8 & 0.000 \\
\hline & 643.2 & 0.535 & 645.0 & 522.738 & 645.0 & 522.557 \\
\hline & 651.5 & 178.322 & 651.5 & 178.357 & 656.3 & 401.179 \\
\hline & 660.4 & 69.223 & 663.2 & 113.778 & 663.2 & 113.833 \\
\hline & 667.6 & 4.794 & 667.6 & 4.842 & 687.4 & 0.005 \\
\hline & 687.4 & 0.005 & 704.3 & 10.637 & 826.4 & 65.423 \\
\hline & 826.4 & 65.446 & 836.6 & 11.944 & 836.6 & 11.939 \\
\hline & 872.1 & 231.755 & 889.4 & 0.000 & 916.0 & 0.000 \\
\hline & 922.7 & 97.460 & 922.7 & 97.475 & 955.5 & 38.728 \\
\hline & 955.5 & 38.725 & 964.2 & 0.000 & 977.3 & 122.556 \\
\hline & 977.3 & 122.604 & 988.2 & 0.340 & 988.2 & 0.327 \\
\hline & 992.2 & 197.084 & 1004.5 & 47.167 & 1004.5 & 47.157 \\
\hline & 1008.6 & 157.217 & 1925.2 & 236.917 & 1925.2 & 237.043 \\
\hline & 1930.1 & 24.759 & 1942.6 & 381.722 & 1942.6 & 381.612 \\
\hline & 1949.2 & 96.887 & 1970.4 & 298.015 & 1970.4 & 298.007 \\
\hline & 1979.0 & 115.306 & 2002.7 & 257.393 & 3467.4 & 1.108 \\
\hline & 3468.6 & 13.286 & 3468.6 & 13.292 & 3481.1 & 4.916 \\
\hline & 3482.0 & 18.072 & 3482.0 & 18.073 & 3541.8 & 12.185 \\
\hline & 3544.0 & 22.176 & 3544.0 & 22.169 & 3568.3 & 18.806 \\
\hline \multirow[t]{13}{*}[\mathrm{HGaNH}]{$_{10} \mathrm{C}_{2 \mathrm{~h}}$} & 62.8 & 0.818 & 84.5 & 0.073 & 89.9 & 0.000 \\
\hline & 94.8 & 0.155 & 105.5 & 0.000 & 108.8 & 0.000 \\
\hline & 117.2 & 0.000 & 125.8 & 0.149 & 138.6 & 0.053 \\
\hline & 141.8 & 0.000 & 151.7 & 0.000 & 152.4 & 0.000 \\
\hline & 166.6 & 0.029 & 190.5 & 0.004 & 205.2 & 0.000 \\
\hline & 205.7 & 1.183 & 209.9 & 0.000 & 218.9 & 0.037 \\
\hline & 227.3 & 0.000 & 230.4 & 0.228 & 248.0 & 0.000 \\
\hline & 249.5 & 0.901 & 274.5 & 0.860 & 274.9 & 0.000 \\
\hline & 354.4 & 0.000 & 359.0 & 0.322 & 366.7 & 0.589 \\
\hline & 372.2 & 0.000 & 377.5 & 0.000 & 384.1 & 0.000 \\
\hline & 385.5 & 3.482 & 392.1 & 6.188 & 401.5 & 2.656 \\
\hline & 422.5 & 0.327 & 436.2 & 0.000 & 438.4 & 0.000 \\
\hline & 447.0 & 0.372 & 452.0 & 0.000 & 462.3 & 0.000 \\
\hline
\end{tabular}


(C) 2004 American Chemical Society, J. Am. Chem. Soc., Timoshkin ja0400483 Supporting Info Page 5

\begin{tabular}{|c|c|c|c|c|c|c|}
\hline & 490.8 & 16.439 & 494.7 & 0.000 & 496.5 & 0.000 \\
\hline & 501.7 & 2.800 & 515.2 & 49.123 & 521.5 & 65.386 \\
\hline & 538.7 & 0.000 & 541.2 & 8.593 & 543.9 & 0.000 \\
\hline & 553.6 & 0.000 & 557.7 & 0.000 & 558.9 & 0.654 \\
\hline & 571.1 & 23.155 & 573.5 & 0.000 & 580.4 & 0.000 \\
\hline & 602.2 & 56.621 & 620.3 & 0.000 & 625.4 & 230.106 \\
\hline & 632.5 & 182.027 & 635.2 & 0.000 & 636.7 & 0.000 \\
\hline & 639.2 & 1124.132 & 644.6 & 522.972 & 650.9 & 470.554 \\
\hline & 657.6 & 0.000 & 661.5 & 869.462 & 663.2 & 0.000 \\
\hline & 668.0 & 28.137 & 677.7 & 0.000 & 683.1 & 106.250 \\
\hline & 684.0 & 0.000 & 696.5 & 0.000 & 701.0 & 12.915 \\
\hline & 708.5 & 44.564 & 712.5 & 0.000 & 817.5 & 0.000 \\
\hline & 819.7 & 201.724 & 820.4 & 34.393 & 821.1 & 0.000 \\
\hline & 890.0 & 84.025 & 893.0 & 0.000 & 909.8 & 0.000 \\
\hline & 914.7 & 185.719 & 954.0 & 0.000 & 958.0 & 180.631 \\
\hline & 969.9 & 261.680 & 975.5 & 0.000 & 977.2 & 0.000 \\
\hline & 981.6 & 2.830 & 994.8 & 166.010 & 999.4 & 0.000 \\
\hline & 1018.5 & 0.000 & 1025.7 & 88.023 & 1040.7 & 86.229 \\
\hline & 1045.4 & 0.000 & 1913.1 & 465.327 & 1913.9 & 0.000 \\
\hline & 1943.8 & 0.000 & 1944.1 & 2.087 & 1953.5 & 834.111 \\
\hline & 1955.4 & 0.000 & 1978.6 & 0.000 & 1979.1 & 469.559 \\
\hline & 1982.5 & 607.908 & 1985.9 & 0.000 & 3447.8 & 27.671 \\
\hline & 3447.8 & 0.000 & 3475.9 & 0.000 & 3475.9 & 2.199 \\
\hline & 3476.7 & 50.840 & 3476.8 & 0.000 & 3533.4 & 45.426 \\
\hline & 3533.5 & 0.000 & 3534.4 & 0.000 & 3534.4 & 34.039 \\
\hline$[\mathrm{HGaNH}]_{11}$ & 83.0 & 0.169 & 94.0 & 0.006 & 98.0 & 0.208 \\
\hline & 99.3 & 0.148 & 100.0 & 0.163 & 106.5 & 0.006 \\
\hline & 109.0 & 0.003 & 114.3 & 0.142 & 115.2 & 0.152 \\
\hline & 123.5 & 0.091 & 132.9 & 0.038 & 139.9 & 0.083 \\
\hline & 146.8 & 0.011 & 159.0 & 0.010 & 185.8 & 0.021 \\
\hline & 187.8 & 0.055 & 202.4 & 0.373 & 211.3 & 0.509 \\
\hline & 214.3 & 0.176 & 217.9 & 0.428 & 217.9 & 0.001 \\
\hline & 221.5 & 0.368 & 226.7 & 0.413 & 241.0 & 1.038 \\
\hline & 252.8 & 0.881 & 266.3 & 1.022 & 273.0 & 0.441 \\
\hline & 341.9 & 0.120 & 342.0 & 1.113 & 351.9 & 0.382 \\
\hline & 356.0 & 0.988 & 369.2 & 0.488 & 369.4 & 0.070 \\
\hline & 387.8 & 1.303 & 403.3 & 2.618 & 404.6 & 4.236 \\
\hline & 413.8 & 0.372 & 415.5 & 1.199 & 424.8 & 1.541 \\
\hline & 429.2 & 0.001 & 442.2 & 0.520 & 460.4 & 2.700 \\
\hline & 464.0 & 5.388 & 476.9 & 0.908 & 478.7 & 19.883 \\
\hline & 488.6 & 6.695 & 494.8 & 7.682 & 502.3 & 4.036 \\
\hline & 518.7 & 8.708 & 524.4 & 0.879 & 538.9 & 40.346 \\
\hline & 540.4 & 10.619 & 549.7 & 6.181 & 551.8 & 19.144 \\
\hline & 555.4 & 0.995 & 559.1 & 1.475 & 572.5 & 7.849 \\
\hline & 574.8 & 12.454 & 575.8 & 9.407 & 584.9 & 13.321 \\
\hline & 610.6 & 7.489 & 621.7 & 1.834 & 622.4 & 15.111 \\
\hline & 631.8 & 64.889 & 639.4 & 263.202 & 643.3 & 498.160 \\
\hline & 646.1 & 179.602 & 652.6 & 426.206 & 655.3 & 1127.105 \\
\hline & 661.3 & 965.073 & 664.0 & 66.319 & 668.2 & 7.172 \\
\hline
\end{tabular}


(C) 2004 American Chemical Society, J. Am. Chem. Soc., Timoshkin ja0400483 Supporting Info Page 6

\begin{tabular}{|c|c|c|c|c|c|c|}
\hline & 675.2 & 27.846 & 675.3 & 150.880 & 676.1 & 89.777 \\
\hline & 684.1 & 17.858 & 702.0 & 58.361 & 709.1 & 54.055 \\
\hline & 711.1 & 38.540 & 711.8 & 82.632 & 732.1 & 38.578 \\
\hline & 736.0 & 28.796 & 823.7 & 17.106 & 825.2 & 117.887 \\
\hline & 886.8 & 11.928 & 896.3 & 11.238 & 900.0 & 5.913 \\
\hline & 904.4 & 23.494 & 913.8 & 57.333 & 918.7 & 97.434 \\
\hline & 921.5 & 86.122 & 926.4 & 125.793 & 954.1 & 38.916 \\
\hline & 957.0 & 130.892 & 1002.2 & 0.341 & 1014.8 & 101.132 \\
\hline & 1020.7 & 86.737 & 1021.0 & 27.680 & 1031.1 & 60.805 \\
\hline & 1038.8 & 71.316 & 1039.4 & 109.573 & 1052.8 & 2.795 \\
\hline & 1056.5 & 47.055 & 1062.9 & 25.940 & 1905.7 & 247.936 \\
\hline & 1934.7 & 132.446 & 1937.2 & 30.784 & 1941.8 & 181.942 \\
\hline & 1942.1 & 4.220 & 1943.7 & 273.706 & 1946.7 & 349.851 \\
\hline & 1948.0 & 871.493 & 1953.8 & 148.434 & 1975.2 & 389.377 \\
\hline & 1977.5 & 139.953 & 3440.0 & 16.053 & 3463.5 & 4.741 \\
\hline & 3463.6 & 27.893 & 3470.1 & 2.837 & 3470.3 & 28.804 \\
\hline & 3477.8 & 18.239 & 3482.6 & 26.604 & 3482.7 & 12.484 \\
\hline \multirow{32}{*}[\mathrm{HGaNH}]{$_{12}$} & 3484.0 & 15.787 & 3531.5 & 25.093 & 3531.6 & 14.094 \\
\hline & 97.1 & 0.000 & 97.2 & 0.000 & 97.4 & 0.000 \\
\hline & 98.4 & 0.000 & 99.0 & 0.000 & 112.2 & 0.502 \\
\hline & 112.7 & 0.491 & 113.4 & 0.491 & 114.9 & 0.000 \\
\hline & 115.2 & 0.000 & 128.8 & 0.000 & 129.5 & 0.000 \\
\hline & 130.2 & 0.000 & 142.8 & 0.003 & 142.9 & 0.006 \\
\hline & 143.0 & 0.006 & 178.4 & 0.000 & 209.7 & 0.000 \\
\hline & 212.1 & 0.351 & 212.4 & 0.343 & 212.6 & 0.313 \\
\hline & 215.8 & 0.000 & 216.1 & 0.000 & 216.5 & 0.000 \\
\hline & 219.4 & 0.000 & 240.5 & 1.741 & 240.5 & 1.712 \\
\hline & 240.8 & 1.703 & 263.6 & 0.000 & 263.9 & 0.000 \\
\hline & 337.7 & 0.000 & 337.9 & 0.000 & 338.8 & 0.000 \\
\hline & 347.0 & 1.456 & 347.1 & 1.570 & 347.9 & 1.487 \\
\hline & 399.6 & 0.000 & 400.4 & 0.000 & 410.9 & 0.001 \\
\hline & 411.2 & 0.006 & 418.6 & 0.000 & 419.7 & 0.000 \\
\hline & 420.0 & 0.060 & 420.4 & 0.000 & 431.4 & 11.735 \\
\hline & 432.1 & 11.583 & 432.8 & 11.890 & 476.6 & 0.000 \\
\hline & 477.0 & 0.000 & 477.8 & 0.000 & 487.1 & 1.773 \\
\hline & 487.5 & 2.032 & 488.6 & 1.797 & 506.4 & 0.000 \\
\hline & 534.5 & 0.000 & 549.1 & 0.000 & 550.2 & 0.000 \\
\hline & 551.0 & 0.000 & 556.4 & 56.009 & 556.9 & 57.927 \\
\hline & 557.7 & 62.455 & 564.8 & 0.136 & 566.9 & 0.408 \\
\hline & 581.1 & 0.000 & 582.5 & 0.000 & 583.6 & 0.000 \\
\hline & 594.5 & 0.421 & 640.9 & 359.469 & 642.8 & 0.000 \\
\hline & 642.9 & 388.301 & 643.7 & 336.099 & 644.8 & 0.000 \\
\hline & 645.2 & 0.000 & 663.5 & 834.692 & 664.0 & 881.193 \\
\hline & 665.1 & 909.152 & 671.3 & 0.000 & 672.6 & 0.000 \\
\hline & 675.5 & 0.000 & 676.5 & 0.000 & 676.8 & 0.000 \\
\hline & 679.7 & 293.680 & 680.3 & 247.753 & 680.7 & 294.219 \\
\hline & 734.5 & 102.708 & 735.0 & 101.532 & 735.3 & 0.000 \\
\hline & 735.9 & 105.603 & 736.3 & 0.000 & 740.7 & 0.000 \\
\hline & 891.8 & 0.052 & 893.5 & 0.586 & 895.5 & 0.000 \\
\hline
\end{tabular}


(C) 2004 American Chemical Society, J. Am. Chem. Soc., Timoshkin ja0400483 Supporting Info Page 7

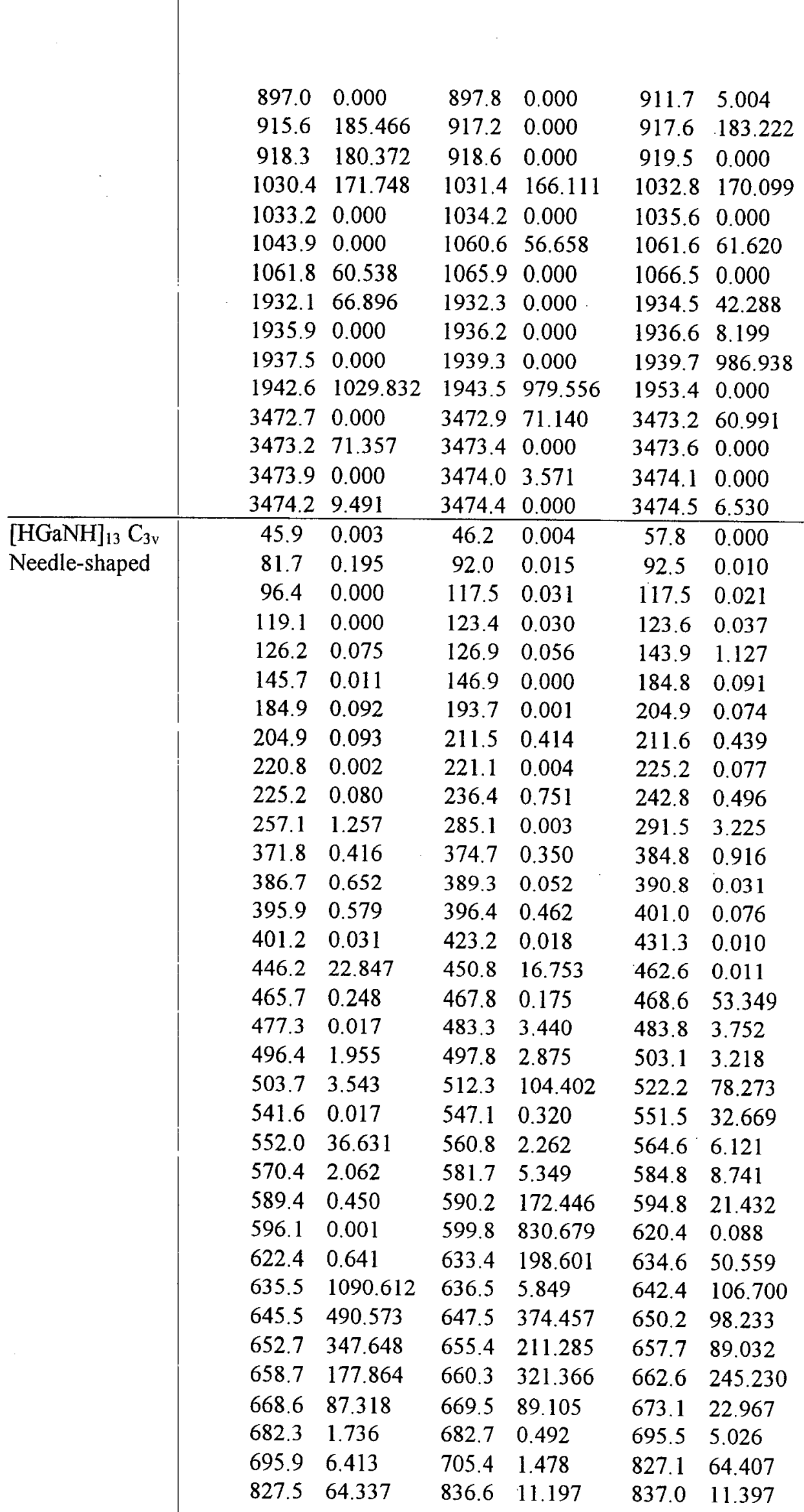


(C) 2004 American Chemical Society, J. Am. Chem. Soc., Timoshkin ja0400483 Supporting Info Page 8

\begin{tabular}{|c|c|c|c|c|c|c|}
\hline & 872.4 & 236.581 & 886.0 & 0.701 & 917.1 & 0.070 \\
\hline & 918.4 & 98.589 & 921.4 & 98.929 & 956.9 & 46.804 \\
\hline & 959.0 & 44.274 & 963.4 & 0.758 & 968.8 & 13.083 \\
\hline & 973.0 & 27.177 & 984.3 & 130.043 & 985.3 & 118.339 \\
\hline & 986.8 & 7.574 & 987.3 & 24.414 & 988.3 & 12.429 \\
\hline & 992.5 & 272.456 & 1001.8 & 288.038 & 1004.6 & 41.008 \\
\hline & 1005.6 & 48.298 & 1008.5 & 50.481 & 1018.8 & 39.913 \\
\hline & 1019.5 & 41.104 & 1907.3 & 241.146 & 1909.1 & 248.217 \\
\hline & 1914.5 & 37.794 & 1929.6 & 256.385 & 1933.4 & 264.803 \\
\hline & 1938.1 & 41.776 & 1949.7 & 369.743 & 1954.7 & 377.810 \\
\hline & 1959.9 & 119.230 & 1974.1 & 277.079 & 1978.4 & 285.501 \\
\hline & 1985.6 & 135.593 & 2003.2 & 281.554 & 3460.3 & 7.728 \\
\hline & 3461.5 & 17.295 & 3461.5 & 17.391 & 3468.8 & 1.652 \\
\hline & 3469.9 & 13.557 & 3470.2 & 13.249 & 3482.5 & 6.126 \\
\hline & 3483.4 & 18.886 & 3483.5 & 18.797 & 3541.4 & 13.443 \\
\hline & 3543.5 & 22.009 & 3543.7 & 22.038 & 3567.9 & 20.289 \\
\hline$[\mathrm{HGaNH}]_{13} \mathrm{C}_{3 \mathrm{v}}$ & 71.3 & 0.476 & 71.6 & 0.509 & 74.6 & 0.013 \\
\hline & 96.1 & 0.080 & 96.2 & 0.049 & 98.5 & 0.095 \\
\hline & 100.0 & 0.081 & 100.6 & 0.060 & 102.9 & 0.010 \\
\hline & 102.9 & 0.010 & 117.7 & 0.193 & 118.6 & 0.210 \\
\hline & 119,9 & 0.000 & 140.0 & 0.003 & 150.2 & 0.018 \\
\hline & 158.6 & 0.007 & 158.8 & 0.011 & 162.8 & 0.000 \\
\hline & 186.2 & 0.485 & 192.7 & 0.026 & 192.9 & 0.024 \\
\hline & 196.9 & 0.375 & 210.5 & 0.039 & 210.7 & 0.037 \\
\hline & 217.0 & 0.831 & 220.1 & 0.001 & 220.3 & 0.001 \\
\hline & 222.2 & 0.000 & 242.2 & 0.272 & 242.5 & 0.259 \\
\hline & 271.4 & 0.842 & 271.5 & 0.817 & 276.3 & 0.001 \\
\hline & 325.0 & 1.128 & 338.3 & 2.424 & 339.5 & 2.420 \\
\hline & 363.7 & 0.014 & 367.5 & 1.290 & 368.7 & 1.519 \\
\hline & 374.1 & 0.311 & 384.9 & 1.812 & 385.5 & 1.536 \\
\hline & 388.9 & 2.249 & 402.5 & 4.382 & 403.9 & 4.870 \\
\hline & 418.2 & 0.030 & 436.8 & 0.019 & 438.3 & 0.606 \\
\hline & 439.9 & 0.874 & 471.8 & 0.029 & 472.9 & 0.032 \\
\hline & 486.1 & 0.230 & 488.2 & 0.824 & 492.9 & 0.391 \\
\hline & 501.4 & 18.736 & 502.9 & 19.018 & 507.8 & 4.528 \\
\hline & 510.5 & 1.008 & 513.8 & 54.363 & 517.3 & 0.218 \\
\hline & 532.0 & 20.708 & 542.7 & 0.008 & 547.4 & 0.766 \\
\hline & 547.7 & 0.137 & 553.7 & 0.078 & 555.5 & 0.241 \\
\hline & 571.3 & 1.325 & 572.6 & 0.695 & 579.4 & 47.654 \\
\hline & 580.7 & 0.516 & 593.3 & 31.035 & 593.8 & 24.344 \\
\hline & 619.0 & 6.568 & 620.5 & 83.318 & 622.5 & 78.468 \\
\hline & 629.0 & 84.610 & 629.2 & 52.551 & 632.3 & 164.537 \\
\hline & 638.1 & 307.061 & 639.0 & 290.342 & 642.4 & 740.208 \\
\hline & 652.6 & 732.889 & 653.5 & 1340.677 & 657.9 & 547.147 \\
\hline & 672.4 & 168.667 & 674.6 & 153.532 & 686.1 & 20.164 \\
\hline & 687.1 & 60.483 & 688.2 & 1.443 & 689.2 & 13.862 \\
\hline & 692.2 & 28.624 & 695.2 & 1.597 & 702.7 & 40.802 \\
\hline & 703.6 & 38.888 & 706.0 & 0.155 & 717.4 & 71.073 \\
\hline & 718.2 & 29.128 & 718.7 & 0.928 & 822.0 & 46.275 \\
\hline
\end{tabular}


(C) 2004 American Chemical Society, J. Am. Chem. Soc., Timoshkin ja0400483 Supporting Info Page 9

\begin{tabular}{|c|c|c|c|c|c|c|}
\hline & 828.2 & 14.981 & 829.7 & 123.243 & 883.8 & 0.991 \\
\hline & 894.2 & 70.015 & 894.9 & 77.061 & 914.1 & 20.285 \\
\hline & 914.5 & 24.252 & 926.4 & 204.980 & 950.3 & 2.530 \\
\hline & 957.6 & 160.936 & 958.6 & 170.670 & 969.7 & 0.539 \\
\hline & 977.5 & 20.293 & 979.6 & 20.993 & 999.3 & 13.776 \\
\hline & 1001.5 & 129.513 & 1004.3 & 108.904 & 1026.0 & 19.820 \\
\hline & 1027.4 & 15.489 & 1036.2 & 68.620 & 1064.1 & 70.595 \\
\hline & 1065.3 & 71.253 & 1071.1 & 17.981 & 1073.8 & 23.495 \\
\hline & 1088.8 & 4.548 & 1878.0 & 234.430 & 1907.3 & 47.193 \\
\hline & 1907.7 & 47.865 & 1915.0 & 574.137 & 1946.3 & 2.273 \\
\hline & 1948.5 & 0.094 & 1948.5 & 7.387 & 1957.2 & 497.725 \\
\hline & 1957.5 & 500.979 & 1961.1 & 279.737 & 1975.6 & 381.280 \\
\hline & 1977.2 & 291.007 & 1981.5 & 186.787 & 3419.4 & 10.858 \\
\hline & 3429.6 & 34.032 & 3430.2 & 3.528 & 3430.3 & 3.650 \\
\hline & 3480.2 & 8.214 & 3480.3 & 6.565 & 3480.8 & 1.842 \\
\hline & 3481.1 & 11.210 & 3481.5 & 34.684 & 3481.7 & 34.713 \\
\hline & 3534.3 & 24.784 & 3534.4 & 19.521 & 3535.1 & 21.640 \\
\hline$[\mathrm{HGaNH}]_{14} \mathrm{C}_{2 \mathrm{~h}}$ & 41.7 & 0.833 & 55.0 & 0.053 & 60.9 & 0.068 \\
\hline & 81.2 & 0.000 & 89.0 & 0.000 & 92.0 & 0.000 \\
\hline & 92.9 & 0.000 & 94.5 & 0.000 & 105.7 & 0.054 \\
\hline & 113.4 & 0.000 & 114.4 & 0.002 & 127.9 & 0.269 \\
\hline & 133.6 & 0.000 & 138.5 & 0.018 & 140.6 & 0.000 \\
\hline & 141.2 & 0.000 & 144.8 & 0.032 & 148.0 & 0.165 \\
\hline & 154.1 & 0.000 & 168.9 & 0.009 & 173.0 & 0.000 \\
\hline & 180.6 & 0.030 & 203.1 & 0.000 & 207.9 & 0.000 \\
\hline & 209.6 & 0.025 & 209.8 & 0.786 & 218.9 & 0.000 \\
\hline & 224.7 & 1.192 & 235.8 & 1.470 & 238.5 & 0.000 \\
\hline & 240.6 & 0.000 & 245.3 & 0.060 & 250.3 & 0.000 \\
\hline & 251.5 & 0.307 & 274.2 & 1.277 & 274.7 & 0.000 \\
\hline & 355.9 & 0.000 & 356.4 & 0.043 & 365.9 & 0.000 \\
\hline & 366.1 & 0.204 & 367.7 & 0.000 & 372.1 & 0.269 \\
\hline & 376.6 & 0.793 & 381.9 & 0.000 & 385.7 & 2.992 \\
\hline & 390.7 & 0.000 & 395.3 & 0.000 & 400.6 & 1.487 \\
\hline & 422.9 & 11.041 & 439.8 & 0.000 & 440.4 & 0.786 \\
\hline & 441.9 & 0.000 & 445.7 & 0.671 & 456.4 & 0.000 \\
\hline & 470.6 & 19.385 & 472.9 & 0.000 & 487.0 & 3.855 \\
\hline & 488.1 & 0.000 & 492.1 & 0.000 & 496.4 & 0.527 \\
\hline & 498.0 & 0.049 & 502.3 & 0.000 & 509.1 & 0.000 \\
\hline & 523.0 & 194.280 & 528.5 & 0.000 & 532.9 & 0.000 \\
\hline & 534.1 & 22.258 & 547.4 & 3.376 & 553.4 & 0.000 \\
\hline & 558.3 & 0.000 & 558.8 & 45.232 & 562.0 & 0.000 \\
\hline & 562.2 & 4.172 & 580.3 & 0.000 & 585.6 & 10.196 \\
\hline & 588.2 & 239.161 & 597.2 & 0.000 & 604.0 & 0.000 \\
\hline & 610.5 & 149.849 & 624.5 & 220.796 & 625.5 & 2319.727 \\
\hline & 625.7 & 0.000 & 630.4 & 324.196 & 631.4 & 0.000 \\
\hline & 632.9 & 0.000 & 636.2 & 0.000 & 637.1 & 211.124 \\
\hline & 639.0 & 24.819 & 645.2 & 0.000 & 648.2 & 22.186 \\
\hline & 658.2 & 0.000 & 658.4 & 566.528 & 663.5 & 1030.140 \\
\hline & 666.6 & 0.000 & 671.3 & 0.000 & 672.7 & 28.414 \\
\hline
\end{tabular}


(C) 2004 American Chemical Society, J. Am. Chem. Soc., Timoshkin ja0400483 Supporting Info Page 10

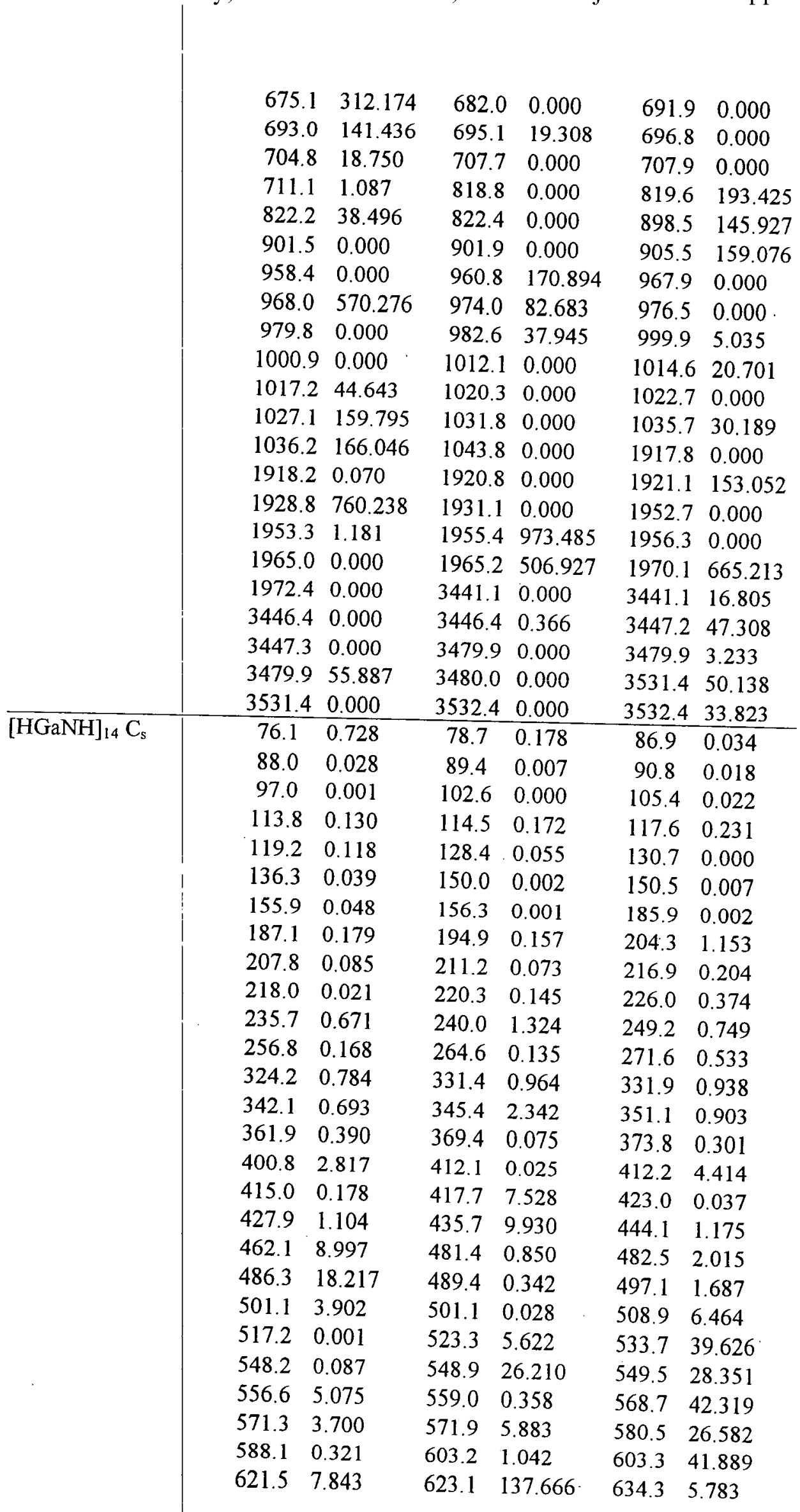


(C) 2004 American Chemical Society, J. Am. Chem. Soc., Timoshkin ja0400483 Supporting Info Page 11

\begin{tabular}{|c|c|c|c|c|c|c|}
\hline & 635.3 & 58.503 & 640.0 & 223.807 & 640.1 & 1.744 \\
\hline & 643.3 & 283.034 & 646.8 & 482.600 & 654.3 & 1265.213 \\
\hline & 658.7 & 1106.164 & 660.6 & 738.450 & 668.7 & 64.443 \\
\hline & 669.5 & 81.337 & 670.3 & 220.949 & 679.5 & 186.944 \\
\hline & 680.4 & 286.629 & 681.8 & 0.048 & 684.6 & 215.725 \\
\hline & 686.7 & 84.664 & 690.6 & 11.038 & 692.6 & 5.618 \\
\hline & 693.9 & 4.199 & 711.1 & 76.580 & 712.0 & 13.721 \\
\hline & 731.6 & 35.254 & 737.0 & 60.842 & 739.1 & 6.906 \\
\hline & 742.2 & 39.107 & 821.8 & 66.457 & 885.0 & 1.113 \\
\hline & 893.1 & 1.671 & 897.7 & 35.355 & 901.6 & 74.257 \\
\hline & 905.4 & 26.840 & 910.8 & 15.730 & 915.7 & 140.552 \\
\hline & 920.8 & 2.217 & 922.9 & 58.141 & 930.2 & 150.030 \\
\hline & 957.5 & 99.630 & 1002.5 & 0.139 & 1005.4 & 1.710 \\
\hline & 1013.5 & 7.414 & 1015.3 & 271.265 & 1024.6 & 99.842 \\
\hline & 1029.8 & 0.376 & 1033.8 & 91.033 & 1038.6 & 84.128 \\
\hline & 1044.3 & 0.547 & 1050.5 & 34.065 & 1058.5 & 7.857 \\
\hline & 1064.7 & 61.097 & 1066.6 & 30.514 & 1066.6 & 83.683 \\
\hline & 1082.9 & 21.000 & 1086.3 & 4.813 & 1890.4 & 223.482 \\
\hline & 1901.3 & 74.252 & 1905.0 & 325.761 & 1935.6 & 24.157 \\
\hline & 1938.6 & 17.575 & 1940.3 & 43.260 & 1940.9 & 12.841 \\
\hline & 1941.6 & 418.636 & 1943.3 & 288.510 & 1945.3 & 283.450 \\
\hline & 1949.9 & 631.230 & 1951.6 & 778.517 & 1956.8 & 58.818 \\
\hline & 1982.4 & 270.269 & 3421.0 & 12.849 & 3426.9 & 22.861 \\
\hline & 3427.5 & 6.880 & 3464.6 & 17.290 & 3467.2 & 33.568 \\
\hline & 3467.6 & 12.613 & 3468.4 & 21.443 & 3468.4 & 1.746 \\
\hline & 3470.1 & 19.485 & 3470.7 & 21.112 & 3478.9 & 19.315 \\
\hline & 3478.9 & 26.925 & 3479.4 & 10.944 & 3534.5 & 22.667 \\
\hline$[\mathrm{HGaNH}]_{15} \mathrm{C}_{3 \mathrm{~h}}$ & 77.7 & 0.893 & 84.0 & 0.026 & 84.0 & 0.026 \\
\hline & 86.6 & 0.000 & 86.6 & 0.000 & 95.3 & 0.000 \\
\hline & 97.2 & 0.170 & 97.2 & 0.170 & 105.3 & 0.000 \\
\hline & 105.3 & 0.000 & 116.1 & 0.000 & 116.1 & 0.000 \\
\hline & 116.8 & 0.146 & 121.3 & 0.000 & 126.0 & 0.277 \\
\hline & 126.0 & 0.277 & 139.2 & 0.093 & 142.2 & 0.000 \\
\hline & 142.2 & 0.000 & 149.4 & 0.004 & 149.4 & 0.004 \\
\hline & 156.6 & 0.000 & 180.4 & 0.000 & 191.9 & 0.000 \\
\hline & 200.5 & 0.045 & 206.0 & 1.503 & 206.0 & 1.503 \\
\hline & 208.4 & 0.000 & 209.5 & 0.188 & 216.6 & 0.000 \\
\hline & 216.6 & 0.000 & 227.4 & 0.276 & 227.4 & 0.277 \\
\hline & 242.1 & 1.861 & 244.1 & 0.000 & 256.8 & 0.426 \\
\hline & 256.8 & 0.426 & 260.0 & 0.000 & 260.0 & 0.000 \\
\hline & 326.4 & 0.645 & 326.4 & 0.645 & 329.8 & 0.000 \\
\hline & 330.8 & 0.378 & 332.8 & 0.000 & 332.8 & 0.000 \\
\hline & 350.4 & 2.705 & 350.4 & 2.703 & 358.2 & 0.000 \\
\hline & 396.2 & $0.000^{\circ}$ & 396.2 & 0.000 & 414.5 & 0.000 \\
\hline & 414.5 & 0.000 & 416.2 & 0.020 & 421.0 & 8.895 \\
\hline & 421.0 & 8.893 & 431.5 & 0.000 & 432.1 & 3.673 \\
\hline & 432.1 & 3.673 & 451.3 & 38.311 & 475.4 & 1.043 \\
\hline & 478.9 & 0.000 & 478.9 & 0.000 & 487.0 & 0.000 \\
\hline & 500.1 & 0.000 & 502.7 & 0.101 & 502.7 & 0.100 \\
\hline
\end{tabular}


(C) 2004 American Chemical Society, J. Am. Chem. Soc., Timoshkin ja0400483 Supporting Info Page 12

\begin{tabular}{|c|c|c|c|c|c|c|}
\hline & 514.7 & 0.000 & 514.7 & 0.000 & 519.0 & 0.000 \\
\hline & 522.7 & 1.277 & 538.6 & 51.632 & 538.6 & 51.631 \\
\hline & 546.6 & 33.529 & 552.8 & 0.000 & 552.8 & 0.000 \\
\hline & 563.2 & 0.000 & 569.2 & 0.000 & 569.2 & 0.000 \\
\hline & 575.9 & 25.855 & 575.9 & 25.861 & 583.7 & 25.385 \\
\hline & 586.0 & 0.000 & 617.9 & 0.000 & 617.9 & 0.000 \\
\hline & 632.0 & 109.399 & 634.0 & 0.000 & 637.6 & 220.847 \\
\hline & 638.3 & 122.930 & 638.3 & 122.943 & 640.5 & 0.000 \\
\hline & 640.5 & 0.000 & 656.6 & 758.309 & 656.6 & 758.252 \\
\hline & 661.2 & 2072.927 & 662.0 & 0.000 & 670.6 & 0.000 \\
\hline & 670.6 & 0.000 & 674.5 & 753.215 & 674.5 & 753.229 \\
\hline & 681.5 & 0.483 & 687.0 & 208.402 & 687.0 & 208.400 \\
\hline & 687.3 & 0.000 & 687.7 & 0.000 & 687.7 & 0.000 \\
\hline & 692.4 & 41.411 & 692.4 & 41.406 & 697.1 & 0.000 \\
\hline & 736.8 & 0.000 & 736.8 & 0.000 & 738.8 & 73.520 \\
\hline & 738.8 & 73.518 & 738.9 & 39.438 & 741.6 & 0.000 \\
\hline & 892.2 & 0.000 & 892.2 & 0.000 & 897.3 & 16.956 \\
\hline & 897.3 & 16.966 & 901.1 & 48.383 & 908.5 & 0.000 \\
\hline & 913.4 & 0.000 & 913.4 & 0.000 & 914.2 & 184.806 \\
\hline & 920.9 & 0.000 & 925.1 & 156.371 & 925.1 & 156.398 \\
\hline & 1019.3 & 0.000 & 1019.3 & 0.000 & 1022.5 & 246.711 \\
\hline & 1032.9 & 0.000 & 1033.3 & 158.429 & 1033.3 & 158.410 \\
\hline & 1040.3 & 39.929 & 1044.9 & 0.000 & 1044.9 & 0.000 \\
\hline & 1049.7 & 0.000 & 1051.2 & 85.168 & 1059.7 & 0.000 \\
\hline & 1059.7 & 0.000 & 1063.1 & 65.700 & 1063.1 & 65.701 \\
\hline & 1081.4 & 37.198 & 1081.4 & 37.167 & 1084.1 & 0.000 \\
\hline & 1899.1 & 306.626 & 1899.1 & 306.604 & 1899.7 & 0.000 \\
\hline & 1932.0 & 4.005 & 1932.3 & 0.000 & 1932.3 & 0.000 \\
\hline & 1937.6 & 406.137 & 1937.6 & 406.110 & 1938.0 & 0.000 \\
\hline & 1944.7 & 0.000 & 1944.7 & 0.000 & 1945.6 & 611.553 \\
\hline & 1945.6 & 611.524 & 1949.8 & 1110.963 & 1955.7 & 0.000 \\
\hline & 3430.2 & 24.128 & 3430.2 & 24.129 & 3430.3 & 0.000 \\
\hline & 3461.7 & 11.681 & 3461.7 & 0.000 & 3461.7 & 0.000 \\
\hline & 3461.9 & 48.850 & 3461.9 & 48.859 & 3462.0 & 0.000 \\
\hline & 3474.2 & 74.386 & 3474.3 & 0.000 & 3474.7 & 0.000 \\
\hline & 3474.7 & 0.000 & 3474.7 & 22.211 & 3474.7 & 22.216 \\
\hline$[\mathrm{HGaNH}]_{16} \mathrm{C}_{3 \mathrm{v}}$ & 81.0 & 0.000 & 82.7 & 0.093 & 82.7 & 0.093 \\
\hline & 87.7 & 0.034 & 87.7 & 0.034 & 91.4 & 0.275 \\
\hline & 93.9 & 0.564 & 93.9 & 0.565 & 101.4 & 0.000 \\
\hline & 105.3 & 0.032 & 105.3 & 0.031 & 113.7 & 0.000 \\
\hline & 114.9 & 0.034 & 114.9 & 0.034 & 123.2 & 0.000 \\
\hline & 123.2 & 0.000 & 123.2 & 0.361 & 132.8 & 0.000 \\
\hline & 138.6 & 0.190 & 138.6 & 0.190 & 142.3 & 0.045 \\
\hline & 153.6 & 0.008 & 153.6 & 0.008 & 161.8 & 0.095 \\
\hline & 190.2 & 0.053 & 194.5 & 0.001 & 194.5 & 0.001 \\
\hline & 198.6 & 1.151 & 198.6 & 1.151 & 206.9 & 0.000 \\
\hline & 215.2 & 0.273 & 215.2 & 0.273 & 217.6 & 1.587 \\
\hline & 225.0 & 0.222 & 225.0 & 0.222 & 227.2 & 0.001 \\
\hline & 242.6 & 0.274 & 253.1 & 0.427 & 253.1 & 0.427 \\
\hline
\end{tabular}


(C) 2004 American Chemical Society, J. Am. Chem. Soc., Timoshkin ja0400483 Supporting Info Page 13

\begin{tabular}{|c|c|c|c|c|c|}
\hline 3.7 & 0.003 & 260.4 & 0.020 & 260.4 & 0.020 \\
\hline 205 & 0.962 & 320.5 & 0.963 & 324.3 & 0.000 \\
\hline 28.9 & 0.155 & 328.9 & 0.154 & 331.9 & 1.760 \\
\hline 345.7 & 0.478 & 346.9 & 0.264 & 346.9 & 0.264 \\
\hline 48.8 & 2.833 & 390.4 & 0.003 & 390.4 & 0.003 \\
\hline 405.9 & 0.000 & 417.7 & 0.000 & 422.6 & 0.114 \\
\hline 422.6 & 0.113 & 431.4 & 13.188 & 431.4 & 13.188 \\
\hline 433.3 & 24.447 & 443.5 & 12.152 & 443.5 & 12.150 \\
\hline 475.9 & 0.000 & 484.6 & 1.041 & 484.6 & 1.042 \\
\hline 491.3 & 5.039 & 493.9 & 0.166 & 493.9 & 0.166 \\
\hline 503.6 & 0.000 & 509.8 & 0.006 & 516.6 & 0.076 \\
\hline 516.6 & 0.076 & 519.2 & 3.012 & 519.2 & 3.015 \\
\hline 538.2 & 26.017 & 538.2 & 26.008 & 546.0 & 0.000 \\
\hline 548.4 & 87.743 & 557.9 & 2.472 & 557.9 & 2.474 \\
\hline 562.7 & 0.000 & 574.2 & 25.441 & 574.2 & 25.431 \\
\hline 582.5 & 3.772 & 582.5 & 3.772 & 583.2 & 0.000 \\
\hline 621.5 & 14.195 & 621.5 & 14.195 & 626.6 & 2.200 \\
\hline 631.4 & 0.000 & 635.3 & 89.590 & 635.3 & 89.586 \\
\hline 638.2 & 0.306 & 638.2 & 0.306 & 640.8 & 0.000 \\
\hline 646.6 & 782.182 & 656.2 & 0.007 & 656.2 & 0.006 \\
\hline 656.6 & 699.491 & 656.6 & 699.734 & 664.7 & 1241.6 \\
\hline 664.7 & 1241.396 & 673.5 & 985.517 & 677.0 & 57.645 \\
\hline 677.0 & 57.630 & 684.5 & 156.427 & 684.5 & 156.426 \\
\hline 685.7 & 0.000 & 685.8 & 44.285 & 687.4 & 1.082 \\
\hline 687.4 & 1.087 & 694.0 & 337.524 & 697.4 & 11.592 \\
\hline 697.4 & 11.581 & 699.8 & 69.060 & 735.3 & 9.624 \\
\hline 735.3 & 9.626 & 737.2 & 31.526 & 747.5 & 16.892 \\
\hline 747.5 & 16.886 & 749.2 & 34.719 & 894.4 & 0.000 \\
\hline 900.8 & 18.127 & 900.8 & 18.120 & 912.6 & 0.000 \\
\hline 916.0 & 43.823 & 916.0 & 43.877 & 918.4 & 8.854 \\
\hline 918.4 & 8.840 & 926.6 & 185.351 & 931.4 & 0.000 \\
\hline 939.5 & 124.141 & 939.5 & 124.153 & 1019.6 & 55.188 \\
\hline 1019.6 & 55.135 & 1026.7 & 0.000 & 1034.1 & 143.762 \\
\hline 1037.6 & 136.738 & 1037.6 & 136.68 & 1045.6 & 35.258 \\
\hline 1045.6 & 35.292 & 1052.1 & 0.000 & 1052.6 & 0.005 \\
\hline 1052.6 & 0.003 & 1059.4 & 109.088 & 1059.8 & 74.465 \\
\hline 1059.8 & 74.392 & 1065.7 & 10.459 & 1065.7 & 10.578 \\
\hline 1071.0 & 40.758 & 1080.7 & 2.072 & 1080.7 & 2.066 \\
\hline 1084.5 & 23.565 & 1896.3 & 305.169 & 1896.3 & 305.197 \\
\hline 1897.4 & 14.183 & 1908.4 & 217.804 & 1935.8 & 9.055 \\
\hline 1935.8 & 9.070 & 1935.9 & 0.000 & 1938.1 & 27.244 \\
\hline 1938.1 & 27.221 & 1940.2 & 142.757 & 1941.9 & 155.746 \\
\hline 1941.9 & 155.785 & 1944.8 & 954.713 & 1947.0 & 837.657 \\
\hline 1947.0 & 837.728 & 1954.6 & 1.663 & 3427.4 & 22.689 \\
\hline 3427.4 & 22.682 & 3427.5 & 1.229 & 3445.1 & 20.838 \\
\hline 3456.4 & 42.161 & 3456.8 & 6.374 & 3456.8 & 6.374 \\
\hline 3466.5 & 30.345 & 3466.5 & 30.332 & 3466.5 & 31.662 \\
\hline 3467.5 & 11.139 & 3467.5 & 11.132 & 3467.6 & 0.000 \\
\hline 3468.8 & 24.985 & 3468.8 & 24.991 & 3468.9 & 6.762 \\
\hline
\end{tabular}


(C) 2004 American Chemical Society, J. Am. Chem. Soc., Timoshkin ja0400483 Supporting Info Page 14

\begin{tabular}{|c|c|c|c|c|c|c|}
\hline$[\mathrm{HGaNH}]_{16} \mathrm{C}_{3 \mathrm{v}}$ & 33.6 & 0.003 & 33.6 & 0.003 & 48.2 & 0.000 \\
\hline Needle-shaped & 65.7 & 0.024 & 66.2 & 0.020 & 69.3 & 0.050 \\
\hline & 76.8 & 0.000 & 101.4 & 0.000 & 102.0 & 0.003 \\
\hline & 102.1 & 0.000 & 114.3 & 0.007 & 114.5 & 0.010 \\
\hline & 118.0 & 0.001 & 121.8 & 0.039 & 122.0 & 0.046 \\
\hline & 124.7 & 0.836 & 126.6 & 0.230 & 127.3 & 0.255 \\
\hline & 130.6 & 0.112 & 131.2 & 0.040 & 146.1 & 0.006 \\
\hline & 147.3 & 0.004 & 169.5 & 0.072 & 186.4 & 0.141 \\
\hline & 186.6 & 0.142 & 204.7 & 0.590 & 206.8 & 0.088 \\
\hline & 206.9 & 0.118 & 211.9 & 0.632 & 211.9 & 0.670 \\
\hline & 218.5 & 0.000 & 218.6 & 0.000 & 225.3 & 0.081 \\
\hline & 225.7 & 0.089 & 228.8 & 0.031 & 229.1 & 0.032 \\
\hline & 240.9 & 0.850 & 246.2 & 0.743 & 255.7 & 1.079 \\
\hline & 269.4 & 0.808 & 292.4 & 0.141 & 299.1 & 4.279 \\
\hline & 384.0 & 0.503 & 385.1 & 0.525 & 391.8 & 0.730 \\
\hline & 392.0 & 0.510 & 398.9 & 0.190 & 398.9 & 0.164 \\
\hline & 404.1 & 0.378 & 404.3 & 0.487 & 406.0 & 0.340 \\
\hline & 408.4 & 0.436 & 410.5 & 0.086 & 410.9 & 0.000 \\
\hline & 435.8 & 0.003 & 450.7 & 0.009 & 453.8 & 27.982 \\
\hline & 458.4 & 6.423 & 474.0 & 12.603 & 474.3 & 0.002 \\
\hline & 476.8 & 104.756 & 479.6 & 0.091 & 481.2 & 0.005 \\
\hline & 481.9 & 0.129 & 491.6 & 0.000 & 500.6 & 3.029 \\
\hline & 500.9 & 3.504 & 512.3 & 0.528 & 512.4 & 0.765 \\
\hline & 521.5 & 9.112 & 521.6 & 73.920 & 521.9 & 15.622 \\
\hline & 524.5 & 0.215 & 525.1 & 0.001 & 536.6 & 89.178 \\
\hline & 552.2 & 0.090 & 552.7 & 0.145 & 563.0 & 21.439 \\
\hline & 564.3 & 27.539 & 570.9 & 2.437 & 572.8 & 3.801 \\
\hline & 576.5 & 0.119 & 592.6 & 4.763 & 594.0 & 2.513 \\
\hline & 598.2 & 3.329 & 599.2 & 3.505 & 601.4 & 0.029 \\
\hline & 602.2 & 35.539 & 609.5 & 1994.137 & 612.1 & 26.935 \\
\hline & 612.9 & 7.399 & 621.8 & 93.231 & 632.9 & 19.569 \\
\hline & 633.9 & 15.814 & 643.6 & 65.540 & 643.7 & 192.560 \\
\hline & 645.4 & 1396.137 & 646.3 & 0.133 & 654.0 & 131.525 \\
\hline & 654.7 & 75.251 & 655.1 & 86.872 & 655.9 & 14.934 \\
\hline & 658.1 & 551.168 & 658.7 & 555.152 & 665.4 & 321.842 \\
\hline & 665.8 & 0.762 & 669.5 & 267.366 & 670.4 & 261.573 \\
\hline & 670.7 & 110.341 & 677.0 & 0.516 & 677.2 & 1.809 \\
\hline & 681.1 & 224.964 & 681.5 & 232.824 & 684.6 & 23.639 \\
\hline & 689.3 & 2.149 & 692.7 & 7.866 & 693.3 & 4.742 \\
\hline & 700.7 & 13.729 & 700.8 & 15.027 & 708.3 & 4.259 \\
\hline & 708.5 & 4.299 & 727.4 & 22.313 & 819.9 & 73.344 \\
\hline & 820.4 & 73.243 & 832.3 & 12.801 & 832.7 & 13.069 \\
\hline & 867.2 & 257.966 & 887.4 & 0.658 & 916.4 & 0.092 \\
\hline & 919.4 & 104.355 & 922.6 & 104.406 & 956.7 & 62.753 \\
\hline & 958.8 & 60.806 & 968.6 & 0.379 & 978.8 & 26.704 \\
\hline & 982.4 & 51.222 & 984.2 & 15.252 & 986.2 & 24.793 \\
\hline & 986.3 & 5.771 & 992.3 & 135.648 & 993.1 & 99.823 \\
\hline & 994.5 & 5.144 & 994.6 & 21.510 & 996.0 & 5.647 \\
\hline & 1001.9 & 552.708 & 1008.3 & 273.469 & 1010.4 & 72. \\
\hline
\end{tabular}


(C) 2004 American Chemical Society, J. Am. Chem. Soc., Timoshkin ja0400483 Supporting Info Page 15

\begin{tabular}{|c|c|c|c|c|c|c|}
\hline & 1013.1 & 62.839 & 1013.6 & 64.159 & 1018.7 & 76.620 \\
\hline & 1020.9 & 83.002 & 1021.4 & 93.966 & 1026.4 & 0.837 \\
\hline & 1027.5 & 0.170 & 1901.0 & 188.587 & 1902.1 & 195.389 \\
\hline & 1907.0 & 18.588 & 1911.0 & 282.780 & 1913.0 & 283.428 \\
\hline & 1919.6 & 56.693 & 1927.8 & 260.439 & 1931.3 & 258.390 \\
\hline & 1935.7 & 33.550 & 1940.9 & 425.424 & 1945.1 & 420.679 \\
\hline & 1951.0 & 138.870 & 1964.8 & 273.837 & 1969.0 & 282.365 \\
\hline & 1976.3 & 135.772 & 1990.7 & 302.734 & 3462.8 & 7.611 \\
\hline & 3464.0 & 18.170 & 3464.0 & 18.629 & 3465.7 & 10.492 \\
\hline & 3467.0 & 19.311 & 3467.2 & 19.821 & 3467.4 & 1.972 \\
\hline & 3468.6 & 15.133 & 3468.9 & 14.203 & 3485.4 & 7.630 \\
\hline & 3486.3 & 21.739 & 3486.4 & 21.636 & 3560.9 & 15.776 \\
\hline & 3563.1 & 24.769 & 3563.3 & 24.782 & 3597.4 & 25.384 \\
\hline \multirow[t]{36}{*}[\mathrm{HGaNH}]{$_{16} \mathrm{~T}_{\mathrm{d}}$} & 82.4 & 0.000 & 82.4 & 0.000 & 89.6 & 0.000 \\
\hline & 89.6 & 0.000 & 89.6 & 0.000 & 90.9 & 0.327 \\
\hline & 90.9 & 0.327 & 90.9 & 0.327 & 112.2 & 0.000 \\
\hline & 112.2 & 0.000 & 112.2 & 0.000 & 113.0 & 0.000 \\
\hline & 116.5 & 0.000 & 116.5 & 0.000 & 123.1 & 0.367 \\
\hline & 123.1 & 0.367 & 123.1 & 0.367 & 136.0 & 0.000 \\
\hline & 136.0 & 0.000 & 136.0 & 0.000 & 148.7 & 0.000 \\
\hline & 155.3 & 0.049 & 155.3 & 0.049 & 155.3 & 0.049 \\
\hline & 192.7 & 0.000 & 196.6 & 0.000 & 196.6 & 0.000 \\
\hline & 207.8 & 0.000 & 207.8 & 0.000 & 207.8 & 0.000 \\
\hline & 210.3 & 2.317 & 210.3 & 2.317 & 210.3 & 2.317 \\
\hline & 225.2 & 0.060 & 225.2 & 0.060 & 225.2 & 0.060 \\
\hline & 247.0 & 0.000 & 254.6 & 0.173 & 254.6 & 0.173 \\
\hline & 254.6 & 0.173 & 263.5 & 0.000 & 263.5 & 0.000 \\
\hline & 325.5 & 0.000 & 325.5 & 0.000 & 325.5 & 0.000 \\
\hline & 335.1 & 0.023 & 335.1 & 0.023 & 335.1 & 0.023 \\
\hline & 343.3 & 0.229 & 343.3 & 0.229 & 343.3 & 0.229 \\
\hline & 343.5 & 0.000 & 390.1 & 0.000 & 390.1 & 0.000 \\
\hline & 411.0 & 0.000 & 427.2 & 0.000 & 427.2 & 0.000 \\
\hline & 427.2 & 0.000 & 431.3 & 26.885 & 431.3 & 26.886 \\
\hline & 431.3 & 26.886 & 440.5 & 0.000 & 440.5 & 0.000 \\
\hline & 484.3 & 0.000 & 484.3 & 0.000 & 484.3 & 0.000 \\
\hline & 491.2 & 0.000 & 511.0 & 4.513 & 511.0 & 4.513 \\
\hline & 511.0 & 4.513 & 514.4 & 0.000 & 514.4 & 0.000 \\
\hline & 514.4 & 0.000 & 524.7 & 0.000 & 524.7 & 0.000 \\
\hline & 543.7 & 0.000 & 543.7 & 0.000 & 543.7 & 0.000 \\
\hline & 554.5 & 54.381 & 554.5 & 54.381 & 554.5 & 54.381 \\
\hline & 565.9 & 0.000 & 575.0 & 0.000 & 575.0 & 0.000 \\
\hline & 582.6 & 0.000 & 582.6 & 0.000 & 582.6 & 0.000 \\
\hline & 627.0 & 0.713 & 627.0 & 0.713 & 627.0 & 0.713 \\
\hline & 631.2 & 0.000 & 631.2 & 0.000 & 631.2 & 0.000 \\
\hline & 653.5 & 480.958 & 653.5 & 480.964 & 653.5 & 480.956 \\
\hline & 653.5 & 0.000 & 653.5 & 0.000 & 657.1 & 0.000 \\
\hline & 657.1 & 0.000 & 657.1 & 0.000 & 669.6 & 1644.753 \\
\hline & 669.6 & 1644.745 & 669.6 & 1644.752 & 681.7 & 0.000 \\
\hline & 681.7 & 0.000 & 686.6 & 11.388 & 686.6 & 11.387 \\
\hline
\end{tabular}


(C) 2004 American Chemical Society, J. Am. Chem. Soc., Timoshkin ja0400483 Supporting Info Page 16

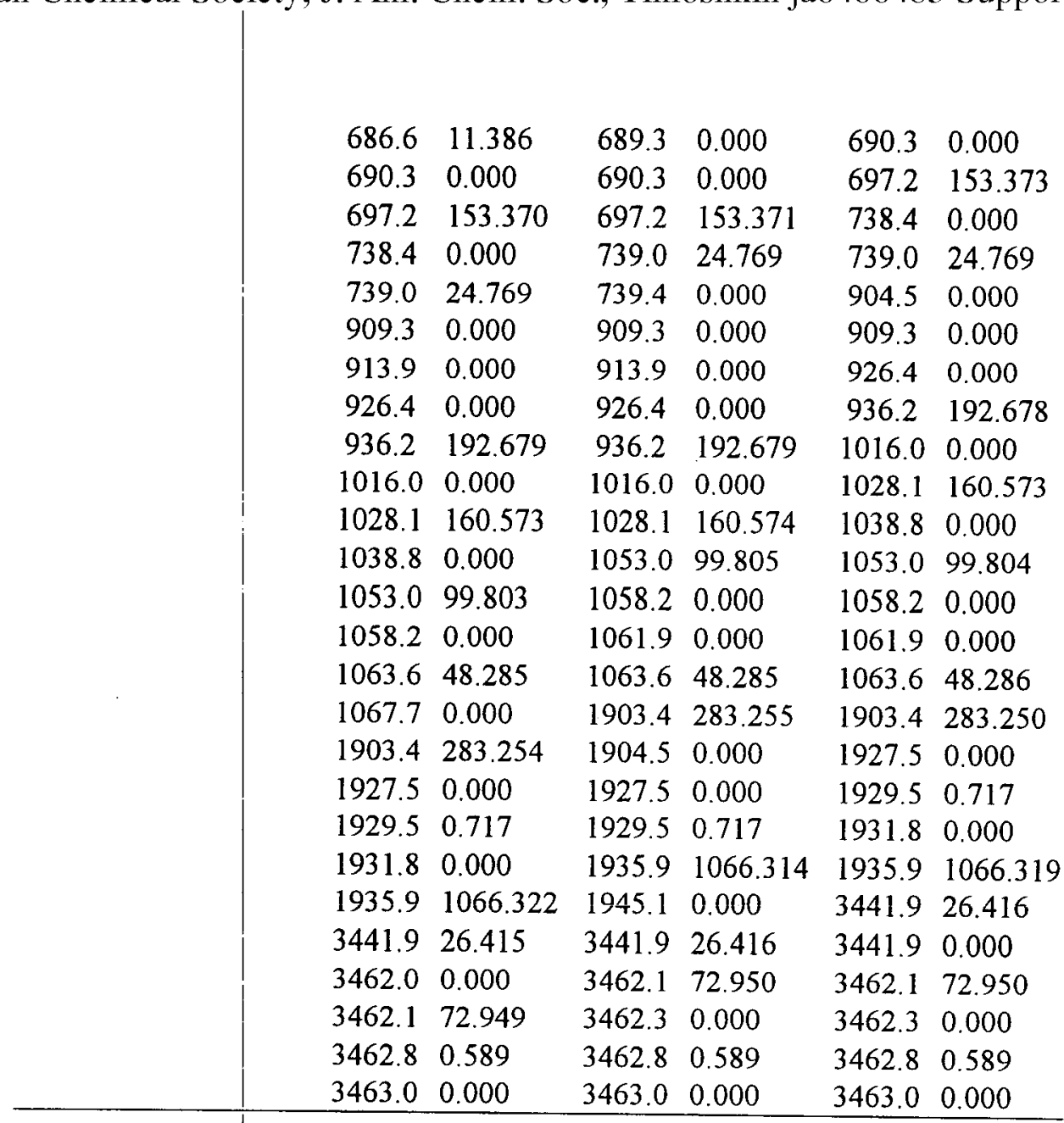


(C) 2004 American Chemical Society, J. Am. Chem. Soc., Timoshkin ja0400483 Supporting Info Page 17

\section{Supplementary Figure Captions.}

Figure 1S. Optimized structural parameters for the investigated $[\mathrm{HGaNH}]_{n}$ compounds. All distances are in Angstroms, all angles in degrees. B3LYP/pVDZ and B3LYP/LANL2DZ $(d, p)$ levels of theory. 
(C) 2004 American Chemical Society, J. Am. Chem. Soc., Timoshkin ja0400483 Supporting Info Page 18

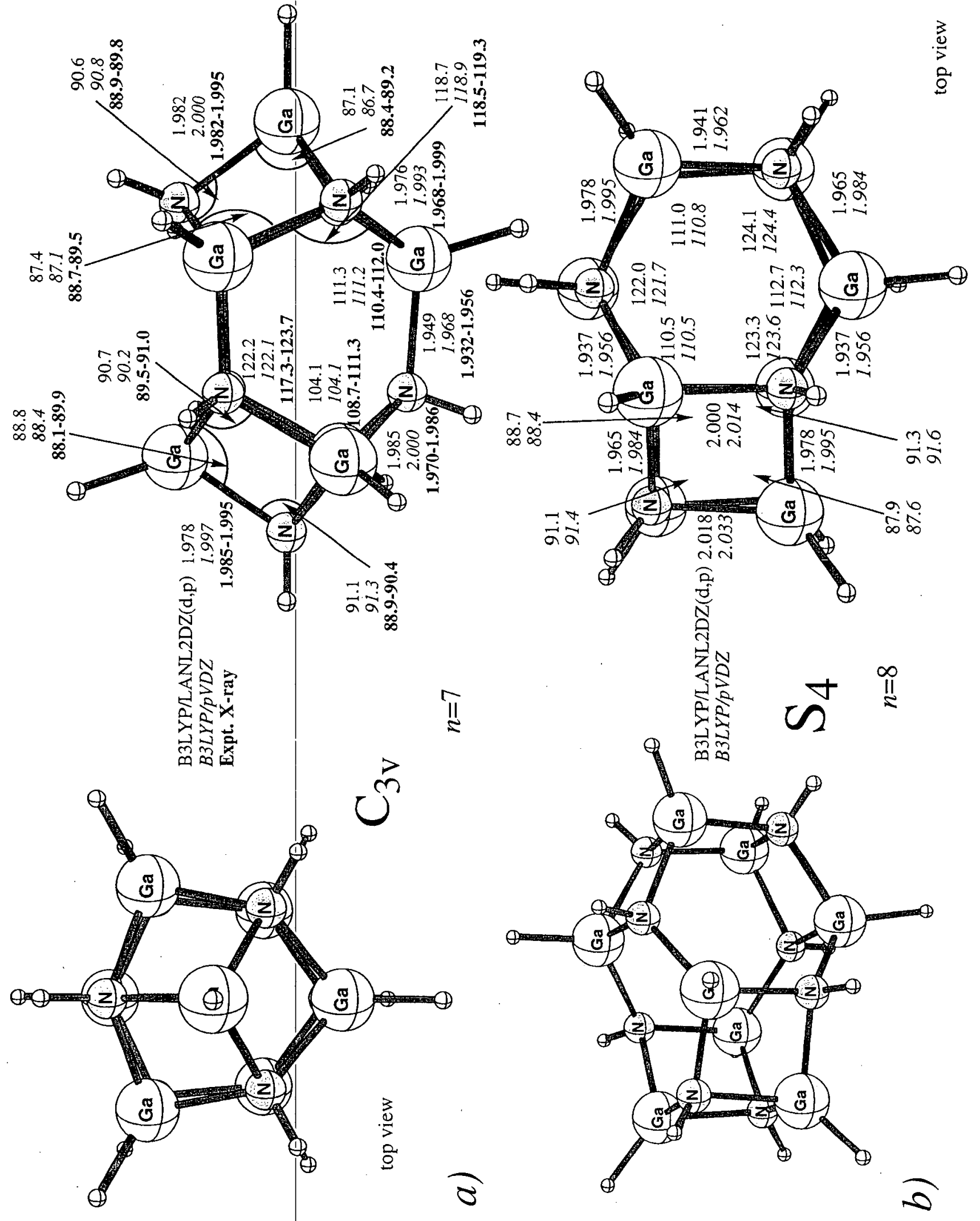


(C) 2004 American Chemical Society, J. Am. Chem. Soc., Timoshkin ja0400483 Supporting Info Page 19

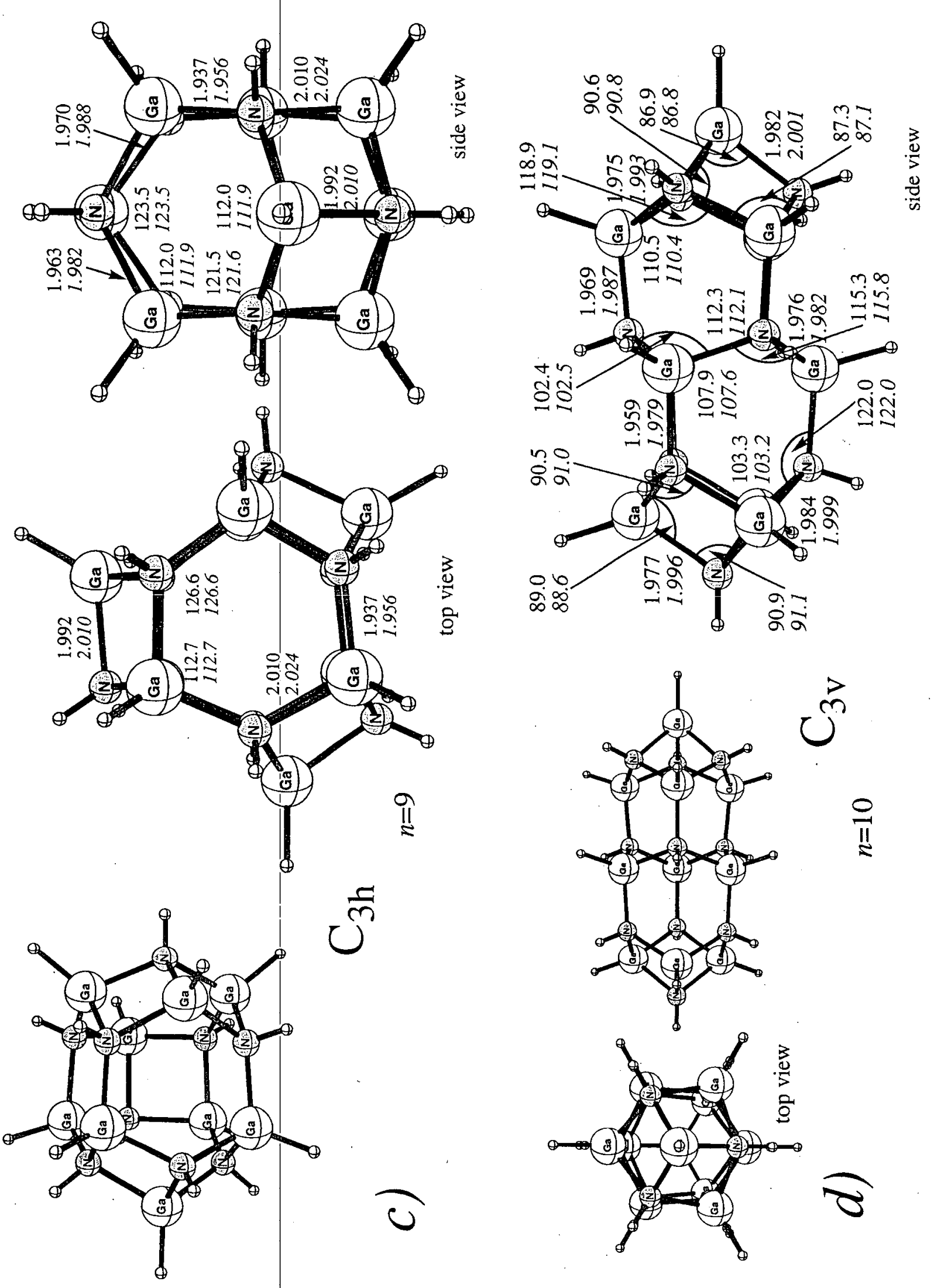


(C) 2004 American Chemical Society, J. Am. Chem. Soc., Timoshkin ja0400483 Supporting Info Page 20

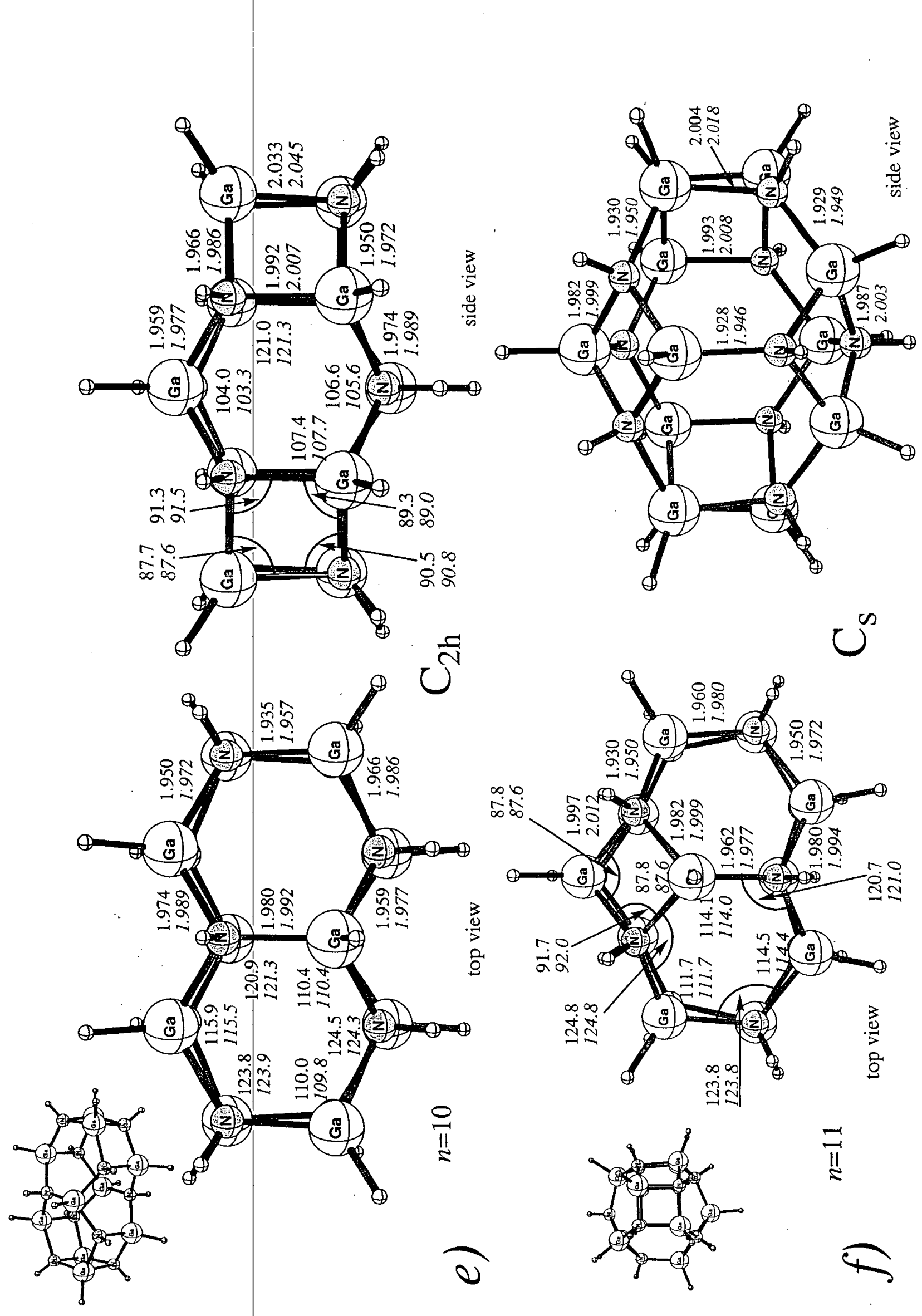


(C) 2004 American Chemical Society, J. Am. Chem. Soc., Timoshkin ja0400483 Supporting Info Page 21

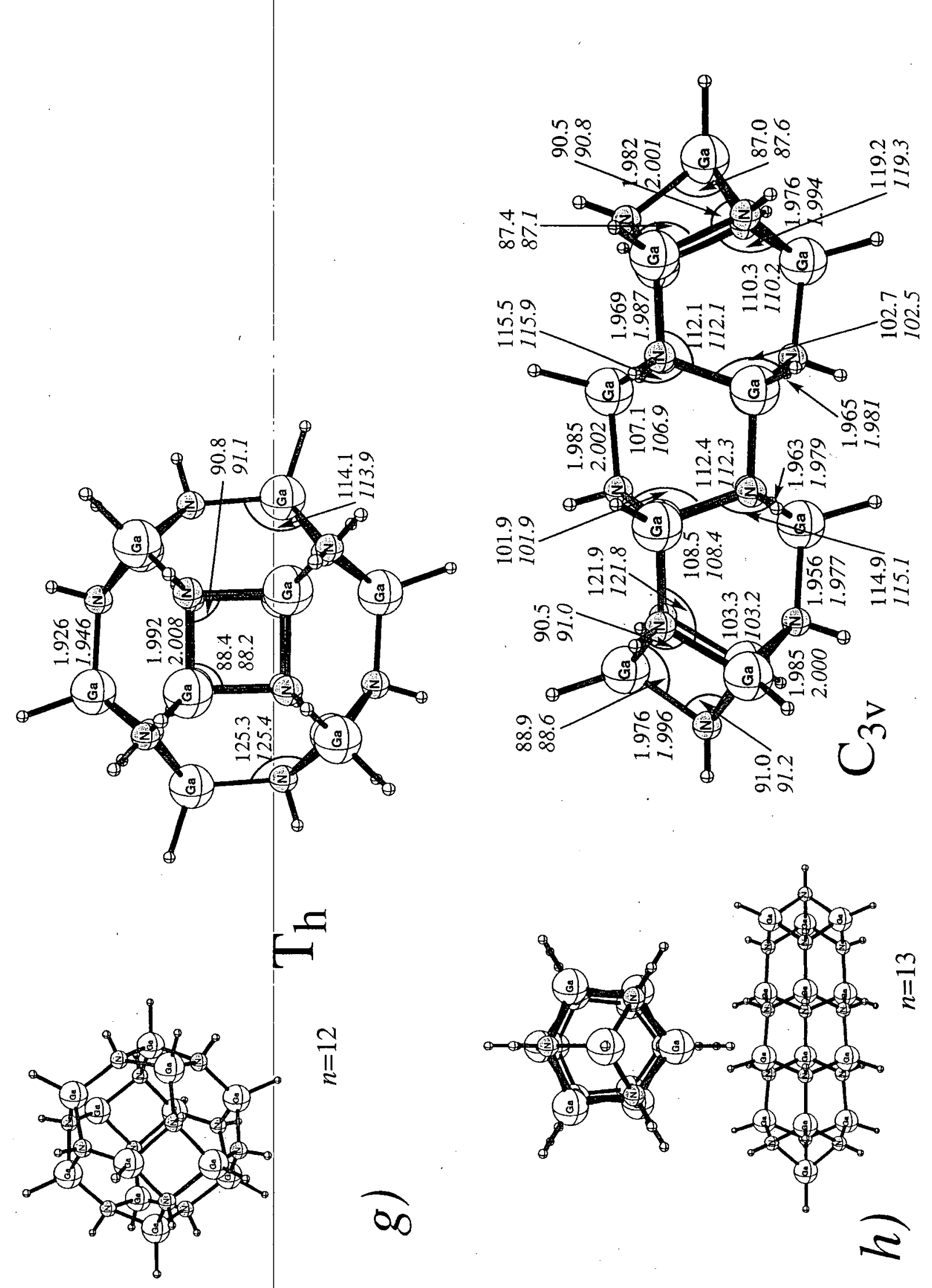


(C) 2004 American Chemical Society, J. Am. Chem. Soc., Timoshkin ja0400483 Supporting Info Page 22

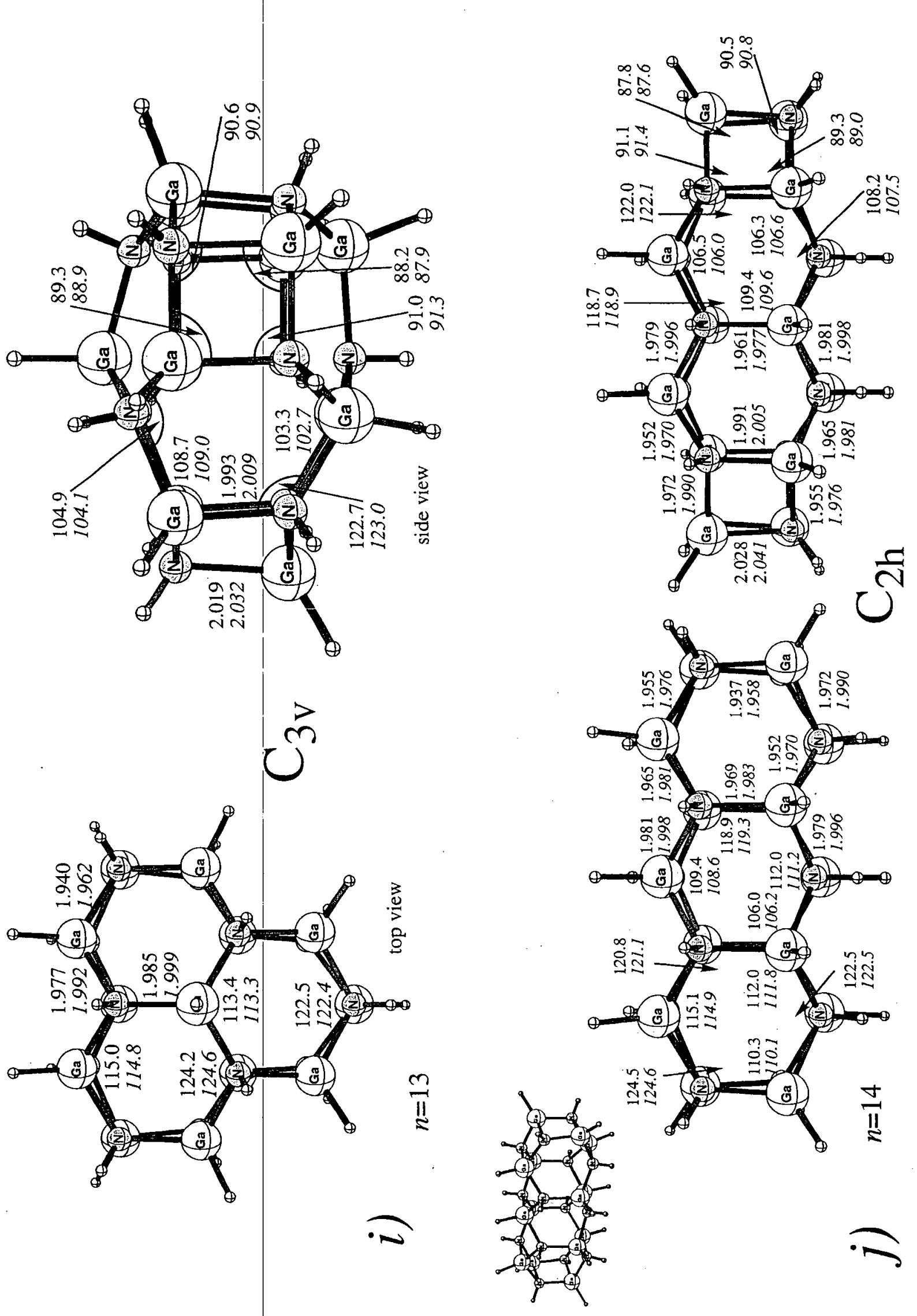


(C) 2004 American Chemical Society, J. Am. Chem. Soc., Timoshkin ja0400483 Supporting Info Page 23

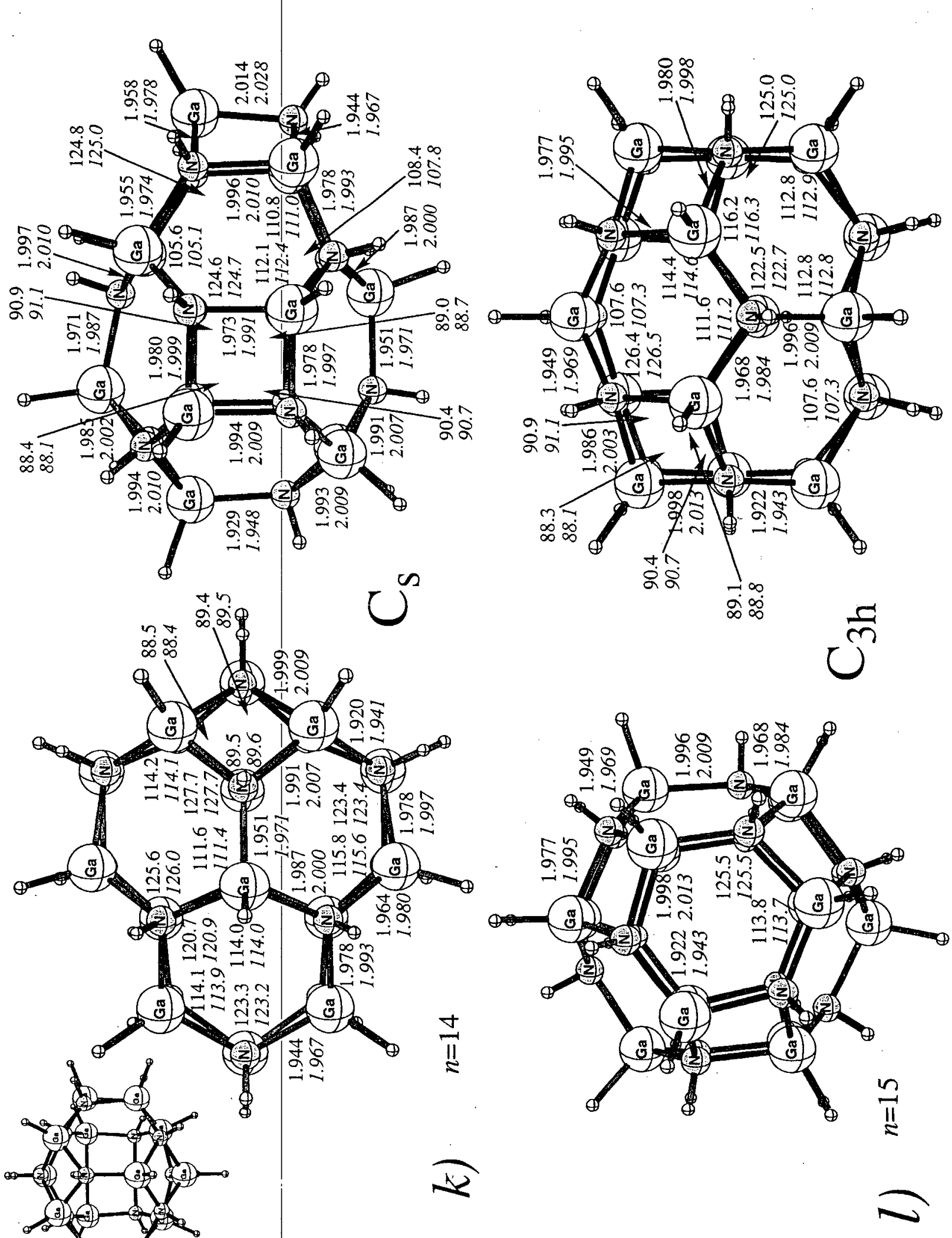


(C) 2004 American Chemical Society, J. Am. Chem. Soc., Timoshkin ja0400483 Supporting Info Page 24

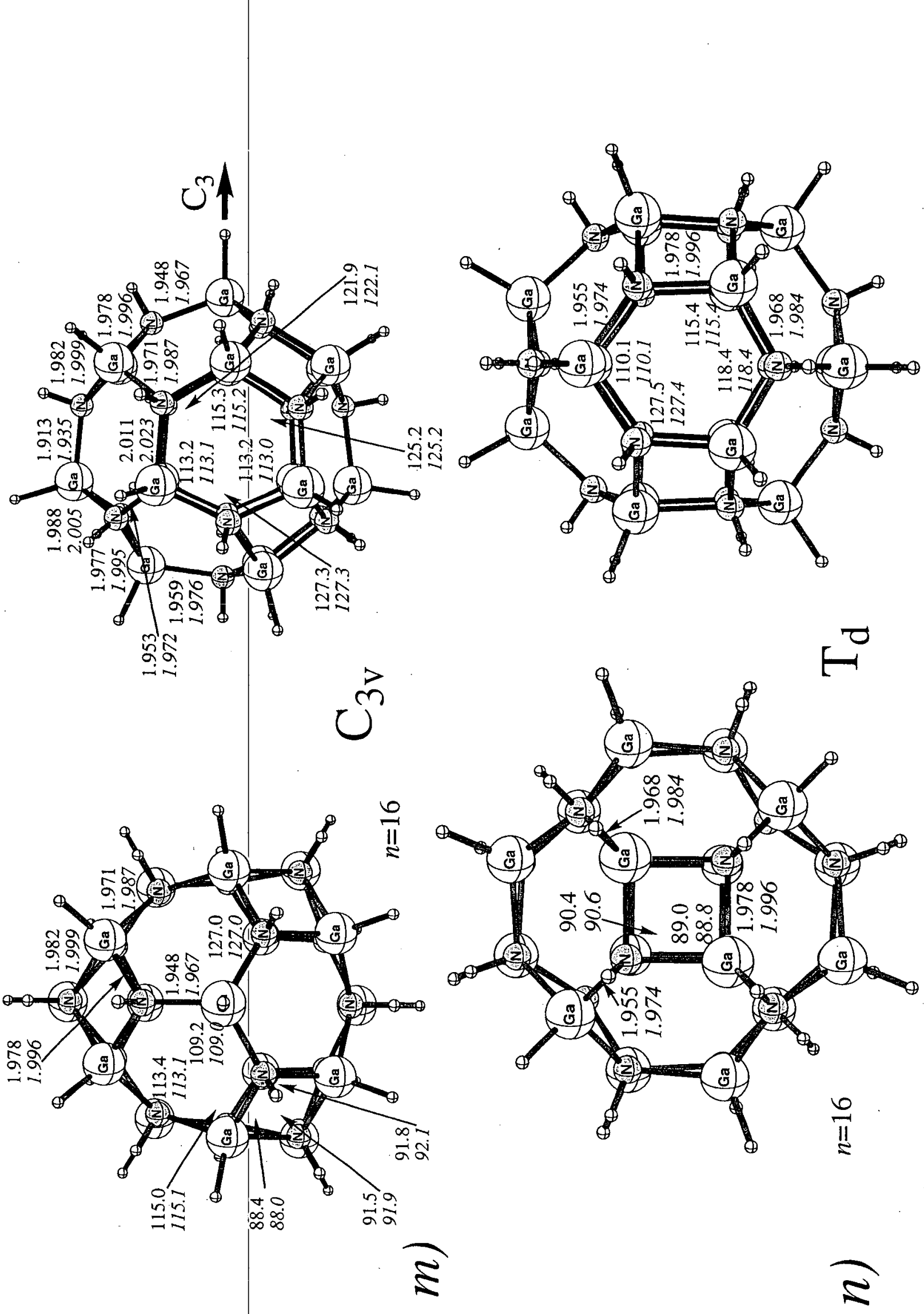


(C) 2004 American Chemical Society, J. Am. Chem. Soc., Timoshkin ja0400483 Supporting Info Page 25

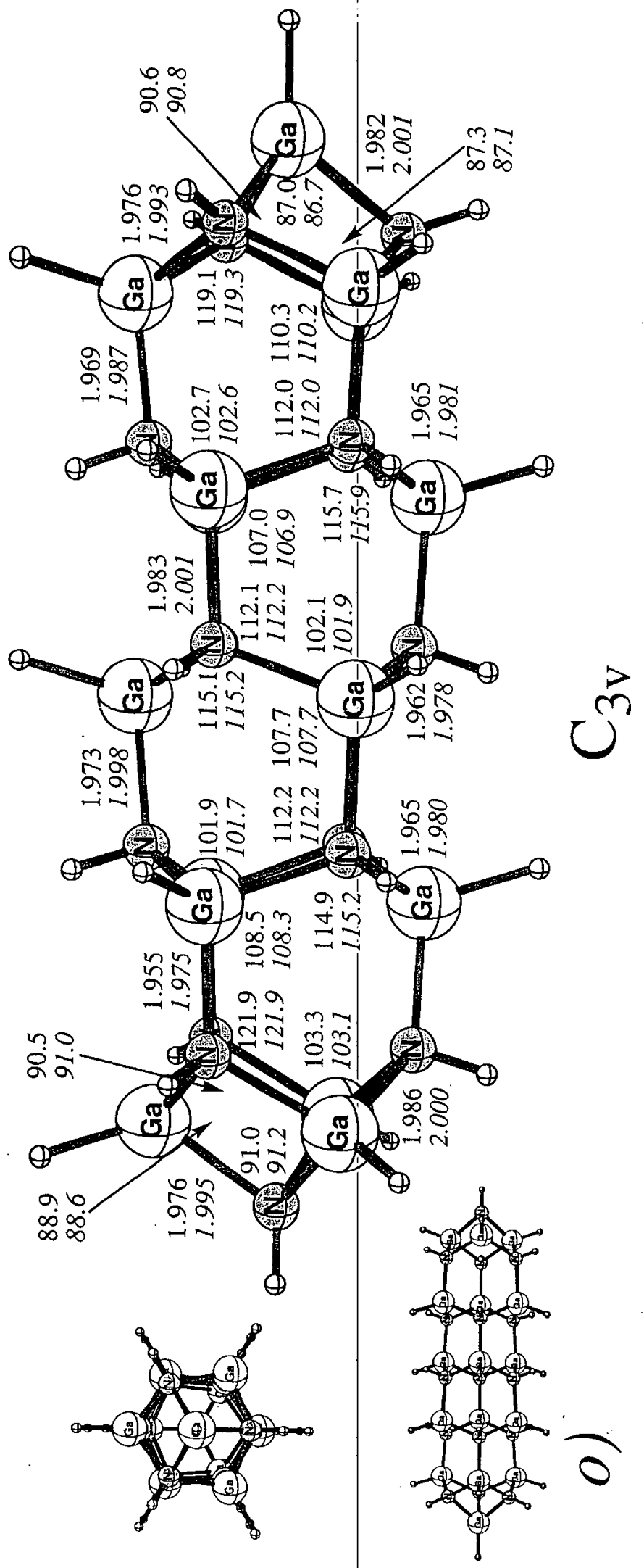

